\title{
Risk- and ambiguity-averse portfolio optimization with quasiconcave utility functionals
}

\author{
Sigrid Källblad ${ }^{1}$
}

Received: 25 November 2014 / Accepted: 15 February 2016 / Published online: 15 March 2017

(C) The Author(s) 2017. This article is published with open access at Springerlink.com

\begin{abstract}
Motivated by recent axiomatic developments, we study the risk- and ambiguity-averse investment problem where trading takes place in continuous time over a fixed finite horizon and terminal payoffs are evaluated according to criteria defined in terms of quasiconcave utility functionals. We extend to the present setting certain existence and duality results established for so-called variational preferences by Schied (Finance Stoch. 11:107-129, 2007). The results are proved by building on existing results for the classical utility maximization problem, combined with a careful analysis of the involved quasiconvex and semicontinuous functions.
\end{abstract}

Keywords Model uncertainty - Ambiguity aversion - Quasiconvex risk measures · Optimal investment $\cdot$ Robust portfolio selection · Duality theory

Mathematics Subject Classification (2010) 49N15 - 62C20 - 91B06 · 91B16 • 91G10 $\cdot 91 \mathrm{G} 80$

JEL Classification $\mathrm{G} 11 \cdot \mathrm{C} 61 \cdot \mathrm{D} 81$

\section{Introduction}

The optimal investment problem of choosing the best way to allocate investors' capital is often formulated as the problem of maximizing, over admissible investment strategies, the expected utility of terminal wealth. The formulation relies on the axiomatic foundation developed by von Neumann and Morgenstern [77] and Savage

\footnotetext{
S. Källblad

sigrid.kaellblad@tuwien.ac.at

1 Institut für Stochastik und Wirtschaftsmathematik, Technische Universität Wien,

Wiedner Hauptstrasse 8-10, 1040 Vienna, Austria
} 
[70]. In continuous-time optimal portfolio selection, the study dates back to the seminal contributions of Merton [60, 61]. In order to formulate the expected utility criterion, the investor needs to specify her preferences via the investment horizon and the utility function, but also her views about the future by providing the probability measure to compute the expectation. The specification of the latter may itself be subject to uncertainty. This is referred to as ambiguity, or Knightian uncertainty in reference to the original contribution of Knight [49]; it has been brought to prominence via the Ellsberg paradox [25].

From a decision-theoretic point of view, the issue was addressed in the seminal work of Gilboa and Schmeidler [35]. They formulated axioms on investors' preferences that account for aversion against both ambiguity and risk. Specifically, within the Anscombe-Aumann model, the axioms of von Neumann and Morgenstern were relaxed in that the axiom of independence was replaced by that of certainty independence. This led to numerical representations of preferences in terms of coherent monetary utility functionals. The robust representation of the latter, see Delbaen [19], then yields for preferences over random variables the representation

$$
X \longmapsto \inf _{\mathbb{Q} \in \mathcal{Q}} \mathrm{E}^{\mathbb{Q}}[U(X)],
$$

where $U$ is a von Neumann-Morgenstern utility function and $\mathcal{Q}$ is a set of probability measures. The latter are referred to as priors and effectively represent potential market models.

The decision-theoretic developments in [35] motivated the study of the risk- and ambiguity-averse investor who trades in continuous time over a fixed finite horizon and evaluates terminal wealth according to the criterion (1.1). The investment problem associated with such multiple-priors preferences has been extensively studied. Stochastic control methods have successfully been applied to obtain explicit solutions for certain utility functions and sets of priors. Specifically, restricting to stochastic factor models, solutions have been obtained in terms of PDEs in [37, 65], and for the non-Markovian case via the use of BSDEs in [63, 68] (see [31] for a full list of references). For general market models and utility functions, we mention in particular the work by Quenez [68] and Schied and Wu [72]. Relying on the results for the classical utility maximization problem by Kramkov and Schachermayer [50, 51], the authors in [68] and [72] establish a dual formulation of the robust problem and prove existence of an optimizer; see also $[9,79]$ for the case including consumption.

The axiomatic results of Gilboa and Schmeidler were later generalized in Maccheroni et al. [57] where the independence axiom was further relaxed. This led to numerical representations in terms of concave monetary utility functionals. Combined with the generalization of the representation results from coherent utility functionals to the concave case, obtained in Föllmer and Schied [29] and Frittelli and RosazzaGianin [34], it implied the numerical representation

$$
X \longmapsto \inf _{\mathbb{Q} \in \mathcal{Q}}\left(\mathrm{E}^{\mathbb{Q}}[U(X)]+\gamma(\mathbb{Q})\right),
$$

for some penalty function $\gamma$. While the multiple-priors setup in (1.1) is a worst-case approach, the appearance of $\gamma$ enables the investor to weight the possible market 
models according to their plausibility, rendering the presentation intuitively appealing.

The investment problem associated with such so-called variational preferences has also been studied. Particular attention has been paid to the case when the penalty function is given by the relative entropy with respect to a reference measure. Such criteria were introduced in the seminal work of Hansen and Sargent [36]; see also [2]. For this choice, the problem is naturally formulated in terms of utility from consumption (or stochastic differential utilities) and the natural tool is the theory of BSDEs. While a systematic study was initiated in [75], these results have been considerably extended in a number of articles; see [8, 15, 26, 40, 56]. For the case of utility from terminal wealth and general variational criteria, stochastic control methods have been successfully applied for the choice of logarithmic utility, for stochastic factor models as well as for the non-Markovian case; see, respectively, [38, 39] and [53]. General existence and duality results have also been established for variational preferences. Specifically, by the use of methods similar to the ones introduced in [68, 72], Schied [71] generalized these results to the concave case; see also [42, 43].

The decision-theoretic results in [35] and [57] were recently yet further extended by developments in Cerreia-Vioglio et al. [11]. Observing that all ambiguity-averse preferences are obtained by weakening the independence axiom (the coordinate independence axiom within the Savage setting), the authors in [11] take this to its extreme by imposing independence only at the level of risk. This yields a numerical representation in terms of quasiconcave utility functionals. Recent advances also yield robust representations of the latter; besides [11, 12], see Drapeau and Kupper [24] and Frittelli and Maggis $[32,33]$. Combined, this leads to the numerical representation of preferences as

$$
X \longmapsto \inf _{\mathbb{Q} \in \mathcal{Q}} G\left(\mathbb{Q}, \mathrm{E}^{\mathbb{Q}}[U(X)]\right),
$$

for some function $G$ which is jointly quasiconvex, lower semicontinuous in its first argument and nondecreasing and right-continuous in its second.

Similarly to the multiple-priors and variational cases, the advances in [11] motivate the study of the associated investment problem. The aim of the present paper is to initiate such a study: within a dominated probabilistic setup, we consider an investor who trades in continuous time over a fixed finite horizon, evaluates terminal wealth according to (1.3), and maximizes this quantity over admissible trading strategies. While the investor's risk aversion is governed by a standard utility function, the ambiguity preferences are thus represented by a quasiconcave utility functional. To the best of our knowledge, this investment problem has not yet been addressed, although we note that the advances in [11] recently motivated the use of quasiconvex risk measures for the Markowitz portfolio problem, see [58, 59], and that quasiconcave utility functions have been previously studied.

The notion is unifying in that all ambiguity-averse preferences, in particular the multiple-priors, entropic and variational ones, are included as special cases. Specifically, while for the variational case, the weighting of potential market models is determined by the penalty function $\gamma$, the function $G$ allows more flexibility. This is of relevance since many interesting and financially significant weighting functions 
do not possess the additive structure characterizing variational preferences. In particular, this more general structure allows a certain dependence of the ambiguity aversion on the investor's wealth level, which is in accordance with the theory of behavioural finance; see [41]. Significant examples also include preferences with a homothetic structure and so-called smooth criteria; the latter were axiomatized in [47] and amount to considering a distribution over possible market models rather than a worst-case approach.

Our first main result establishes the existence of an optimal strategy; notably this result does not require any particular compactness assumptions on the set of priors. Second, we establish certain duality results. The advantages of studying the problem within the dual domain are particularly evident for robust preferences, where the dual problem amounts to the search for a pure infimum, whereas the primal problem features a minimax structure. In particular, this link allows us to obtain a necessary and sufficient condition for the primal problem to admit a saddle point, as well as an alternative route to solving for the optimal strategy. Finally, we establish various properties of the associated value function.

Our results extend those established for variational preferences in [71] to the case of quasiconcave preferences; see $[68,72]$ for the multiple-priors case. Following this previous literature, we prove our results by building on existing results for the classical utility maximization problem. The quasiconcavity as opposed to concavity of the utility functional implies, however, the need for substantial extensions of the arguments in [71]. First, we need throughout to work with quasiconvexity and semicontinuity, as opposed to convexity and continuity, which requires a more elaborate analysis and additional arguments. Moreover, our approach is based on a more careful study of a certain auxiliary investment problem. In particular, the latter enables a further financial interpretation of the resulting minimax measures, as well as alternative proofs for some of the results in the variational case.

The rest of the paper is organized as follows. In Sect. 2, we provide the axiomatic motivation for our choice of criterion and specify the market restrictions and our investment problem. The main results are presented in Sect. 3. The proofs are given in Sect. 4, where we also provide a discussion of how our methods compare to the existing literature.

\section{Ambiguity-averse investment criteria}

We provide the axiomatic foundation for our criterion in Sect. 2.1 and discuss some examples of particular interest in Sect. 2.2. The reader only interested in the main results may pass directly to the problem formulation in Sect. 2.3.

\subsection{Axiomatic motivation and numerical representation of preferences}

The fundamental question of how to characterize and represent well-founded choices is the focus of the decision-theoretic literature. The question is mostly formalized via the study of so-called preference orders: we write $x \succeq y$ if $x$ is preferred to $y$, for $x, y \in \mathcal{X}$, where $\mathcal{X}$ denotes the set of available objects. Further, $\succeq$ is called a 
preorder on $\mathcal{X}$ if it is reflexive and transitive, and a total preorder if it is also complete. ${ }^{1}$ Naturally, we write $x \succ y$ for the negation of $y \succeq x$, and $x \sim y$ if $x \succeq y$ and $y \succeq x$. The use of preference orders is greatly facilitated if the order admits a socalled numerical representation, i.e., a function $F: \mathcal{X} \rightarrow \mathbb{R}$ such that $x \succeq y$ if and only if $F(x) \geq F(y)$. In the seminal work of von Neumann and Morgenstern [77, Sect. I.3.6], natural axioms which (sensible) preorders should satisfy and which imply the existence of such a numerical representation were formulated (see also Savage [70, Chapters 1-5]). Specifically, denoting by $\mathcal{M}_{1, c}$ the set of probability distributions on $\mathbb{R}$ with bounded support (referred to as lotteries) the two main axioms of von Neumann and Morgenstern read as follows:

NM0 (Archimedean axiom) For any triple $\mu \succ \lambda \succ v$ in $\mathcal{M}_{1, c}$, there are $\alpha, \beta \in(0,1)$ such that $\alpha \mu+(1-\alpha) v \succ \lambda \succ \beta \mu+(1-\beta) v$.

NM1 (Independence axiom) For all $\mu, v \in \mathcal{M}_{1, c}$, the relation $\mu \succ v$ implies that $\alpha \mu+(1-\alpha) \lambda \succ \alpha \nu+(1-\alpha) \lambda$ for all $\lambda \in \mathcal{M}_{1, c}$ and $\alpha \in(0,1]$.

It was shown in [77] that these axioms are necessary and sufficient for a total preorder to admit a numerical representation $F(\mu)=\mathfrak{U}(\mu)$, for some affine functional $\mathfrak{U}: \mathcal{M}_{1, c} \rightarrow \mathbb{R}$. Further, given e.g. monotonicity with respect to first order stochastic dominance (see [30, Chapter 2]), this affine representation admits the integral representation

$$
\mathfrak{U}(\mu)=\int U(x) \mu(\mathrm{d} x)
$$

for some function $U: \mathbb{R} \rightarrow \mathbb{R}$. This result forms the axiomatic basis for the problem of maximization of (subjective) expected utility, ${ }^{2}$ which since the seminal contributions of [60] has played a prominent role within mathematical finance; see, among others, [16, 45, 46, 67].

The question of model uncertainty was incorporated in the axiomatic approaches starting with the work of Gilboa and Schmeidler [35], later followed by, among others, Maccheroni et al. [57] and Cerreia-Vioglio et al. [11]; see also [10, 13]. Axioms are then placed on preferences over a set of so-called random lotteries, also referred to as horse acts. To specify this, let $(\Omega, \mathcal{F})$ be a measurable space and consider the class $\tilde{\mathcal{X}}$ of Markov kernels $X(\omega, \mathrm{d} y)$ from $(\Omega, \mathcal{F})$ to $\mathbb{R}$ for which there exists a compact set $K \subset \mathbb{R}$ such that $X(\omega, K)=1$ for all $\omega \in \Omega$. We recall the following axioms from the literature:

A1 (Monotonicity) If $\tilde{Y}(\omega) \succeq \tilde{X}(\omega)$ for all $\omega \in \Omega$, then $\tilde{Y} \succeq \tilde{X}$.

A2 (Uncertainty aversion) If $\tilde{X} \sim \tilde{Y}, \tilde{X}, \tilde{Y} \in \tilde{\mathcal{X}}$, then $\alpha \tilde{X}+(1-\alpha) \tilde{Y} \succeq \tilde{X}$ for all $\alpha \in[0,1]$.

A3 (Archimedean axiom) If $\tilde{X}, \tilde{Y}, \tilde{Z} \in \tilde{\mathcal{X}}$ are such that $\tilde{Z} \succ \tilde{Y} \succ \tilde{X}$, then there are $\alpha, \beta \in[0,1]$ such that $\alpha \tilde{Z}+(1-\alpha) \tilde{X} \succ \tilde{Y} \succ \beta \tilde{Z}+(1-\beta) \tilde{X}$.

\footnotetext{
${ }^{1} \mathrm{~A}$ binary relation $\succeq$ on $\mathcal{X}$ is reflexive if $x \succeq x$, for all $x \in \mathcal{X}$; transitive if $y \succeq x$ and $z \succeq y$ implies $z \succeq x$, for $x, y, z \in \mathcal{X}$; and complete if $x \succeq y$ or $y \succeq x$, for all $x, y \in \mathcal{X}$.

${ }^{2}$ The additional requirements that $\delta_{x} \succ \delta y$, for $x>y$, and that the certain amount $\int x \mu(d x)$ is preferred to the lottery $\mu$, are equivalent to the function $U: \mathbb{R} \rightarrow \mathbb{R}$ being a strictly increasing and concave utility function. The expected utility criterion may also be derived by formulating the axioms directly on the set of random variables; see e.g. [24].
} 
The axioms of monotonicity and uncertainty aversion are the most fundamental axioms for decision making: while the former quantifies that more is better, the latter is due to Schmeidler [73] and formalizes decision makers' preference for randomization. Its interpretation in terms of a negative attitude towards uncertainty dates back to Debreu [18]; see [11] for further discussion. The Archimedean axiom is a continuity axiom. Notably, at the level of acts, we have not included any axiom of independence. Indeed, in order to formulate axioms so as to allow model uncertainty and obtain ambiguity-averse preferences, it is the axiom of independence that needs to be relaxed. While Gilboa and Schmeidler [35] replaced it by so-called certainty independence at the level of acts, this axiom was further weakened by Maccheroni et al. [57] (see Sect. 2.2 below). In Cerreia-Vioglio et al. [11] (see also [24]), it was yet further weakened in that independence was imposed only at the level of risk. Such preferences therefore correspond to the most fundamental class of preferences modelling decision making under model uncertainty.

In order to recall the representation result from [11], let $\mathcal{X}$ be an ordered linear space of bounded functions containing the constants. We then define as follows:

Definition 2.1 A mapping $\phi: \mathcal{X} \rightarrow \overline{\mathbb{R}}$ is a quasiconcave utility functional ${ }^{3}$ if it is monotone and quasiconcave; that is, we have $\phi(X) \geq \phi(Y)$ if $X \succeq Y$, and we have $\phi(\lambda X+(1-\lambda) Y) \geq \min \{\phi(X), \phi(Y)\}$, for $X, Y \in \mathcal{X}$ and $\lambda \in[0,1]$.

The next result is an immediate consequence of Theorem 3 in [11] (see also [24]) and follows by adopting the proof along the lines of Theorem 3.1 in [31].

Theorem 2.2 Consider a total preorder $\succeq$ on $\tilde{\mathcal{X}}$ satisfying axioms A1-A3. Suppose further that the restriction of $\succeq$ to $\mathcal{M}_{1, c}$ satisfies the independence axiom NM1, and that its affine numerical representation $\mathfrak{U}: \mathcal{M}_{1, c} \rightarrow \mathbb{R}$ is of the form (2.1) for a utility function $U: \mathbb{R} \rightarrow \mathbb{R}$ with unbounded range. Then there exists a unique extension of $\mathfrak{U}$ to a numerical representation $\tilde{\mathfrak{U}}: \tilde{\mathcal{X}} \rightarrow \mathbb{R}$ with

$$
\overline{\mathfrak{U}}(\tilde{X})=\phi(\mathfrak{U}(\tilde{X}))=\phi\left(\int U(x) \tilde{X}(\cdot, \mathrm{d} x)\right),
$$

where $\phi$ is a quasiconcave utility functional defined on the space of bounded measurable functions on $(\Omega, \mathcal{F}){ }^{4}$

In order to further specify the numerical representation (2.2), we next discuss robust representations of utility functionals. We do so for the case when $\mathcal{X}$ (in Definition 2.1) is the space of (essentially) bounded random variables; that is, $\mathcal{X}=L^{\infty}$, and the preorder is specified by a.s. dominance, for some given probability space $(\Omega, \mathcal{F}, \mathbb{P})$. For the case of coherent and concave utility functionals, robust representation results were then established in [19] and [29, 34]; see Sect. 2.2 below. These results were recently generalized to the quasiconcave case; we here give a result

\footnotetext{
${ }^{3} \mathrm{~A}$ (quasi)concave utility functional is the negative of a (quasi)convex risk measure, i.e., $\phi$ is a (quasi)concave UF if and only if $\rho=-\phi$ is a (quasi)convex RM.

${ }^{4}$ With the preorder specified by $X \succeq Y$ if $X(\omega) \geq Y(\omega)$ for all $\omega \in \Omega$.
} 
from [24], see, however, also [11, Theorem 7]. To this end, recall that a function is quasiconvex if its lower level sets are convex. ${ }^{5}$ With $\mathcal{M}_{1}(\mathbb{P})$ denoting the set of $(\sigma$-additive) probability measures absolutely continuous with respect to $\mathbb{P}$, we then define as follows:

Definition 2.3 Let $\mathcal{G}$ be the set of functions $G: \mathcal{M}_{1}(\mathbb{P}) \times \mathbb{R} \rightarrow \overline{\mathbb{R}}$ satisfying the following conditions:

(i) $G(\mathbb{Q}, \cdot)$ is nondecreasing and right-continuous; ${ }^{6}$

(ii) $G$ is jointly quasiconvex;

(iii) $G^{-}(\cdot, t)$ is weakly lower semicontinuous, where $G^{-}(\mathbb{Q}, t):=\sup _{s<t} G(\mathbb{Q}, s)$, for $\mathbb{Q} \in \mathcal{M}_{1}(\mathbb{P})$

(iv) $G$ has an asymptotic maximum in the sense that for all $\mathbb{Q}, \overline{\mathbb{Q}} \in \mathcal{M}_{1}(\mathbb{P})$,

$$
A M(G):=\lim _{t \rightarrow \infty} G(\mathbb{Q}, t)=\lim _{t \rightarrow \infty} G(\overline{\mathbb{Q}}, t) .
$$

Theorem 2.4 ([24, Theorem 3.2]) Any $\sigma\left(L^{\infty}, L^{1}\right)$-upper semicontinuous ${ }^{7}$ quasiconcave utility functional $\phi: L^{\infty} \rightarrow \overline{\mathbb{R}}$ admits the representation

$$
\phi(X)=\inf _{\mathbb{Q} \in \mathcal{M}_{1}(\mathbb{P})} G\left(\mathbb{Q}, \mathrm{E}^{\mathbb{Q}}[X]\right)
$$

for some function $G \in \mathcal{G}$. Conversely, for any $G \in \mathcal{G}$, the function $\phi$ defined in (2.3) is an upper semicontinuous quasiconcave utility functional.

For our purposes, we are interested in comparing random variables; specifically, terminal payoffs corresponding to different investment strategies. First, note that a preorder on $\tilde{\mathcal{X}}$ may be restricted to the set of bounded measurable functions $X$ on $(\Omega, \mathcal{F})$ by viewing the latter as elements of $\tilde{\mathcal{X}}$ via their identification with $\delta_{X}$. Combined with Theorem $2.4,(2.2)$ then motivates evaluating random variables according to

$$
X \longmapsto \inf _{\mathbb{Q} \in \mathcal{Q}} G\left(\mathbb{Q}, \mathrm{E}^{\mathbb{Q}}[U(X)]\right)
$$

for some $G \in \mathcal{G}$, with

$$
\mathcal{Q}:=\left\{\mathbb{Q} \in \mathcal{M}_{1}(\mathbb{P}): G(\mathbb{Q}, t)<\infty \text { for some } t>0\right\} .
$$

This is in fact the representation we are interested in. The axioms need, however, to be placed on the class of random lotteries. Due to the nature of those, axioms are thus placed on two levels of uncertainty; similarly, the representation (2.4) admits two levels of uncertainty. It is therefore natural to refer to the lower and upper level as

\footnotetext{
${ }^{5}$ Note that for a given function, say $f: \mathcal{X} \rightarrow \mathbb{R}$, the lower level sets are convex if and only if $f(\lambda x+(1-\lambda) y) \leq \max \{f(x), f(y)\}$ for $x, y \in \mathcal{X}$ and $\lambda \in[0,1]$. A function is quasiconcave if it is the negative of a quasiconvex function; that is, if its upper level sets are convex.

${ }^{6}$ In particular, $G(\mathbb{Q}, \cdot)$ is therefore both quasiconvex and quasiconcave as well as upper semicontinuous.

${ }^{7}$ This holds whenever the utility functional satisfies the Fatou property; see [24].
} 
risk and model uncertainty, respectively. While the axioms at the lower level, and thus the attitude towards risk, render the utility function $U$, it is the axioms at the upper level, and thus the attitude towards ambiguity, that give the function $G$. In effect, the criterion (2.4) evaluates random variables $X$ according to their expected utility under different measures $\mathbb{Q}$, weighted according to their plausibility, with $U$ and $G$, respectively, modelling the risk- and ambiguity-aversion. For a detailed exposition of numerical representations of preferences, we refer to [31, Sect. I.3].

\subsection{Further axioms and significant examples}

Multiple-priors and variational preferences We recall the certainty independence axioms introduced in Gilboa and Schmeidler [35] and Maccheroni et al. [57]:

(Full certainty independence) If $\tilde{X}, \tilde{Y} \in \tilde{\mathcal{X}}$ and $\tilde{X} \succ \tilde{Y}$, then for all $\alpha \in(0,1]$ and $\mu \in \mathcal{M}_{1, c}(\mathbb{R}), \alpha \tilde{X}+(1-\alpha) \mu \succ \alpha \tilde{Y}+(1-\alpha) \mu$.

(Weak certainty independence) If $\tilde{X}, \tilde{Y} \in \tilde{\mathcal{X}}$ and for some $\alpha \in(0,1]$ and $v \in \mathcal{M}_{1, c}(\mathbb{R}), \alpha \tilde{X}+(1-\alpha) v \succ \alpha \tilde{Y}+(1-\alpha) v$, then for all $\mu \in \mathcal{M}_{1, c}(\mathbb{R})$, $\alpha \tilde{X}+(1-\alpha) \mu \succ \alpha \tilde{Y}+(1-\alpha) \mu$.

The results in $[35,57]$ state that if in addition to the axioms listed above, weak (resp. full) certainty independence holds, then the preorder admits the representation (2.2) for a concave (resp. coherent) monetary utility functional; see [31, Theorem 3.1]. Recall that a concave monetary utility functional is a quasiconcave utility functional which is also translation invariant ${ }^{8}$ if it is further positively homogeneous, ${ }^{9}$ it is coherent. For the concave case, representation results akin to Theorem 2.4 were established in [29, 34] (see [19] for the coherent case). Specifically, the function $G \in \mathcal{G}$ then takes the form $G(\mathbb{Q}, t)=t+\gamma(\mathbb{Q})$, where $\gamma$ is the associated penalty function (for the coherent case $\gamma \in\{0, \infty\}$ ), and (2.4) reduces to the variational criterion (1.2). Those preferences therefore correspond to the class of criteria for which the weighting function $G \in \mathcal{G}$ is additive, ${ }^{10}$ and have to date received most attention in the literature on risk- and ambiguity-averse portfolio optimization.

Homothetic preferences It is shown in [57] that a preorder satisfies full certainty independence if and only if it satisfies weak certainty independence along with the following axiom of homotheticity:

(Homotheticity) For all $\tilde{X}, \tilde{Y} \in \tilde{X}, \mu \in \mathcal{M}_{1, c}(\mathbb{R})$ and $\alpha, \beta \in(0,1]$, it holds that $\alpha \tilde{X}+(1-\alpha) \mu \succeq \alpha \tilde{Y}+(1-\alpha) \mu$ implies $\beta \tilde{X}+(1-\beta) \mu \succeq \beta \tilde{Y}+(1-\beta) \mu$.

\footnotetext{
${ }^{8}$ Translation invariance is also referred to as cash additivity and means that for $m \in \mathbb{R}$, $\phi(X+m)=\phi(X)+m$. Any translation invariant quasiconcave utility functional is concave, i.e., $\phi(\lambda X+(1-\lambda) Y) \geq \lambda \phi(X)+(1-\lambda) \phi(Y), \lambda \in[0,1]$; see e.g. [24, Proposition 1.18]. In particular, for risk measures, while diversification is in general (e.g. when only cash subadditivity holds) characterized by quasiconvexity, it is under cash additivity equivalent to convexity.

${ }^{9}$ That is, $\phi(\lambda X)=\lambda \phi(X), \lambda>0$; see [3].

${ }^{10}$ Indeed, also the converse holds true: any quasiconcave utility functional with the property that $G(\mathbb{Q}, t+m)=G(\mathbb{Q}, t)+m$ for $t, m \in \mathbb{R}$ is translation invariant and thus also concave; see [24, Proposition 2.11].
} 
Those two axioms can therefore be seen as symmetric weakenings of the full certainty independence. In particular, preferences satisfying homotheticity (but not necessarily independence) provide an interesting class of preferences: under certain continuity assumptions, a preorder of the form (2.2) satisfies the homotheticity axiom if and only if the corresponding $G$ takes the form

$$
G(\mathbb{Q}, t)= \begin{cases}g_{1}(\mathbb{Q}) h(t), & t \geq 0, \\ g_{2}(\mathbb{Q}) h(t), & t<0,\end{cases}
$$

for functions $g_{1}, g_{2}$ and $h$ with certain properties; we refer to Theorem 26 in [11]. In consequence, the class of preferences satisfying the axiom of homotheticity corresponds to the class of preferences for which $G$ has a multiplicative structure. Preferences of this type have been studied in [14]. We also note that quasiconcave utility functionals corresponding to so-called certainty equivalents and the economic index of riskiness have this multiplicative structure; see [24, Examples 3.3 and 3.4].

Smooth preferences In [47] (see also [76]), so-called smooth criteria are considered. Specifically, by adding to the above list of axioms, they obtain a class of preferences for which the numerical representation (2.4) takes the form

$$
X \longmapsto \psi^{(-1)}\left(\int_{\mathcal{M}_{1}(\mathbb{P})} \psi\left(\mathrm{E}^{\mathbb{Q}}[U(X)]\right) \mu(\mathrm{d} \mathbb{Q})\right),
$$

where $U$ models the risk aversion, $\psi$ is an increasing and concave function modelling the ambiguity aversion, and $\mu$ is a distribution over $\mathcal{M}_{1}(\mathbb{P})$; we refer to [47] for the precise axioms, and to [11] for the specific form of the corresponding $G \in \mathcal{G}$. Rather than taking the (weighted) infimum over possible market models, the decision maker thus considers an average over all models, weighted according to a distribution representing her modelling beliefs. Such criteria formalize an intuitively appealing behaviour. For exponential $\psi$, the smooth criteria are actually of variational form; in general, this is, however, not the case.

\subsection{The risk- and ambiguity-averse investment problem}

We consider a fixed finite horizon $T>0$ and a given filtered probability space $(\Omega, \mathcal{F}, \mathbb{F}, \mathbb{P})$, where $\mathbb{F}=\left(\mathcal{F}_{t}\right)_{t \in[0, T]}$ satisfies the usual conditions and $\mathbb{P}$ is referred to as the reference measure. The market consists of one riskless and $d$ risky assets, denoted by $S^{0}$ and $S=\left(S^{1}, \ldots, S^{d}\right)$. We let $S^{0} \equiv 1$ and suppose that $\left(S_{t}\right)_{t \in[0, T]}$ is a $d$-dimensional semimartingale.

A trading strategy is a $d$-dimensional $\mathbb{F}$-predictable process, $\pi=\left(\pi_{t}\right)_{t \in[0, T]}$, which is $S$-integrable on $[0, T]$. Given an initial capital $x>0$, the associated wealth process $\left(X_{t}^{\pi}\right)_{t \in[0, T]}$ is given by

$$
X_{t}^{\pi}=x+\int_{0}^{t} \pi_{u} \mathrm{~d} S_{u} .
$$

We restrict to trading strategies such that $X_{t}^{\pi} \geq 0 \mathbb{P}$-a.s. for $t \in[0, T]$. The set of such admissible strategies is denoted by $\mathcal{A}(x)$, while $\mathcal{X}(x)$ denotes the associated set 
of wealth processes. We suppose that the market is arbitrage-free with respect to the reference measure $\mathbb{P}$ in the sense that $\mathcal{M} \neq \emptyset$, where $\mathcal{M}$ denotes the set of probability measures equivalent to $\mathbb{P}$ under which each $X \in \mathcal{X}(1)$ is a local martingale; see [21, 22] for the financial significance of this assumption.

We assume the investor to assess utility of terminal wealth according to the riskand ambiguity-averse criterion (2.4). In accordance with the existing literature, we impose the following assumptions on the risk preferences; we denote the set of admissible utility functions by $\mathcal{U}$.

Definition 2.5 $U:(0, \infty) \rightarrow \mathbb{R}$ is an admissible utility function if it is strictly increasing, strictly concave, satisfies the Inada conditions

$$
\lim _{x \rightarrow 0} U^{\prime}(x)=\infty \quad \text { and } \quad \lim _{x \rightarrow \infty} U^{\prime}(x)=0,
$$

and $A E(U)<1$, with the asymptotic elasticity $A E(U):=\lim \sup _{x \rightarrow \infty} \frac{x U^{\prime}(x)}{U(x)}$.

In order to ensure that (2.4) is well defined, we define $\mathrm{E}^{\mathbb{Q}}[F]:=\infty$ whenever $\mathrm{E}^{\mathbb{Q}}\left[F^{+}\right] \wedge \mathrm{E}^{\mathbb{Q}}\left[F^{-}\right]=\infty$. Further, we extend the domain of $G(\mathbb{Q}, \cdot)$ to $\overline{\mathbb{R}}$ by defining $G(\mathbb{Q},-\infty):=-\infty$ and $G(\mathbb{Q}, \infty):=A M(G)$.

Our main problem of study is then the following investment problem:

Problem 2.6 Given $G \in \mathcal{G}$ and $U \in \mathcal{U}$, we consider the risk- and ambiguity-averse investment problem of maximizing the functional (2.4) over admissible terminal payoffs $X_{T}^{\pi}$ with $\pi \in \mathcal{A}(x)$.

The associated value function is given by

$$
u(x):=\sup _{\pi \in \mathcal{A}(x)} \inf _{\mathbb{Q} \in \mathcal{Q}} G\left(\mathbb{Q}, \mathrm{E}^{\mathbb{Q}}\left[U\left(X_{T}^{\pi}\right)\right]\right), \quad x>0,
$$

where we recall that $\mathcal{Q}$ is given in (2.5). As argued above, when $G$ corresponds to a coherent or concave utility functional, the criterion reduces to the multiple-priors and variational preferences studied in, among others, $[68,71,72]$. In the same way as the study of such preferences was motivated by the axiomatic results in $[35,57]$, the study of the more general quasiconcave case relies on the axiomatic extensions in [11]. This evaluation of terminal payoffs is however also highly plausible as it stands, for it provides a natural robust criterion which takes various potential market models into consideration; while the weighting of the models is determined by the penalty function $\gamma$ for the variational case, the function $G$ allows more flexibility. The set of admissible strategies is here defined with respect to the reference measure $\mathbb{P}$, the role of which is to specify the nullsets rather than representing the most likely model. In particular, we consider a dominated setting in which all measures $\mathbb{Q} \in \mathcal{Q}$ are absolutely continuous with respect to $\mathbb{P}$; for ambiguity-averse portfolio optimization with mutually singular measures, see [23, 64].

Remark 2.7 While $U\left(X_{T}\right) \in L^{0}$ for $X \in \mathcal{X}(x)$, the utility functionals defined with respect to $G \in \mathcal{G}$ are defined only on $L^{\infty}$. That is, although the theory of quasiconvex 
risk measures has been extended to more general spaces, we do not restrict the set $\mathcal{G}$ to account for this, but only impose assumptions in order to ensure that (2.7) is well defined. In this aspect, we follow [71]; see, however, [1] for an alternative approach.

We next introduce the auxiliary investment problem, which plays a crucial role in the upcoming analysis. Specifically, given $U \in \mathcal{U}$, let

$$
u_{\mathbb{Q}}(x):=\sup _{\pi \in \mathcal{A}(x)} \mathrm{E}^{\mathbb{Q}}\left[U\left(X_{T}^{\pi}\right)\right], \quad \mathbb{Q} \in \mathcal{M}_{1}(\mathbb{P}) .
$$

While the objective function in (2.8) is defined with respect to the measure $\mathbb{Q}$, the set of admissible strategies is restricted to those admissible with respect to the reference measure $\mathbb{P}$. Hence, while it holds for $\mathbb{Q} \sim \mathbb{P}$ that the auxiliary problem is identical to the classical utility maximization problem under the physical measure $\mathbb{Q}$, this need not be the case for $\mathbb{Q} \ll \mathbb{P}$. In particular, it is a priori not clear whether the market model is arbitrage-free under $\mathbb{Q}$; see Sect. 4.1 for further discussion.

The following standing assumption is imposed throughout to ensure that the investment problem is well posed and non-trivial.

Assumption 2.8 The functions $G \in \mathcal{G}$ and $U \in \mathcal{U}$ are such that there exists $\mathbb{Q} \in \mathcal{Q}$ with

$$
u_{\mathbb{Q}}\left(x_{0}\right)<\infty \text { for some } x_{0}>0 .
$$

Further, for $t \in \mathbb{R}, G(\cdot, t)$ is bounded from below on $\mathcal{Q}$, and for all $x>0$, there exists $\mathbb{Q} \in \mathcal{Q}$ such that $G\left(\mathbb{Q}, u_{\mathbb{Q}}(x)\right)<\infty$.

Lemma 2.9 For $G \in \mathcal{G}$ and $U \in \mathcal{U}$ satisfying Assumption 2.8, $u(x)$ is finite for all $x>0$, and $u:(0, \infty) \rightarrow \mathbb{R}$ is nondecreasing and quasiconcave.

\section{The main results}

\subsection{Existence of an optimal strategy}

First we establish the existence of a solution to Problem 2.6. The question of uniqueness is discussed below.

Theorem 3.1 Let $G \in \mathcal{G}$ and $U \in \mathcal{U}$ be given and suppose that Assumption 2.8 holds. Further, suppose either that (2.9) holds for each $\mathbb{Q} \in \mathcal{Q}$; or that $G(\cdot, t), t \in \mathbb{R}$, is convex, $G$ is finite on $\mathcal{Q} \times \mathbb{R}$, there exists $\mathbb{Q} \in \mathcal{Q}$ with $\mathbb{Q} \sim \mathbb{P}$, and (2.9) holds for each $\mathbb{Q} \in \mathcal{Q}$ with $\mathbb{Q} \sim \mathbb{P}$. Then there exists an optimal investment strategy $\pi^{*} \in \mathcal{A}(x)$ attaining the supremum in (2.7). Furthermore, the value function $u(x), x>0$, is upper semicontinuous.

The above result does not impose any compactness assumptions on $\mathcal{Q}$. Under such general assumptions, it is not clear though whether the ambiguity-averse problem admits a saddle point; we discuss this further in Sect. 3.3. 
We now argue that the finiteness assumptions placed on $u_{\mathbb{Q}}(x), \mathbb{Q} \in \mathcal{Q}$, are natural. To this end, note that the assumption implies that the auxiliary investment problem itself is solvable for each individual model $\mathbb{Q} \in \mathcal{Q}$; see Lemma 4.2 below. Although the auxiliary problem is not the standard one, this might be understood as an absenceof-arbitrage condition ${ }^{11}$ put on each individual model. Recall that the criterion (2.4) emerges due to axioms posed on the preferences, via the robust representation of quasiconcave utility functionals. This motivation per se does not imply that the measures $\mathbb{Q} \in \mathcal{Q}$ satisfy any market related conditions. However, effectively, the ambiguityaverse criterion amounts to taking expected utility with respect to various potential market models, where the contribution from each individual model is weighted according to its plausibility via the function $G \in \mathcal{G}$. It is therefore natural to restrict to functions which only take into consideration measures which constitute sensible market models in that they exclude arbitrage; this is how the assumptions of Theorem 3.1 should be understood. We note that there are stability results in the literature which ensure that if (2.9) holds for some $\mathbb{Q} \in \mathcal{M}_{1}(\mathbb{P})$, then it also holds in a neighbourhood around that $\mathbb{Q}$; see e.g. $[54,78]$. For the case when the infimum and supremum can be interchanged, the set $\mathcal{Q}$ can be restricted to measures for which (2.9) holds without affecting the indirect utility; cf. Remark 4.7.

The assumption that $G(\cdot, t), t \in \mathbb{R}$, is convex is a necessary, but not a sufficient, condition for the associated utility functional to be concave and monetary. The corresponding class of preferences therefore includes, but is not limited to, the variational ones; see Sect. 4 for further comments on the weaker assumptions imposed in this case.

\subsection{The dual problem formulation}

We now turn to the study of the dual version of the risk- and ambiguity-averse investment problem. Specifically, we establish relations between the primal and dual problems and their respective solutions. This yields an alternative approach to solve the problem and a further understanding of the optimal strategy.

For the duality results, we impose the following additional assumption.

Assumption 3.2 The function $G \in \mathcal{G}$ is jointly lower semicontinuous, and the level sets $\mathcal{Q}_{t}(c):=\{\mathbb{Q} \in \mathcal{Q}: G(\mathbb{Q}, t) \leq c\}, t \in \mathbb{R}, c \geq 0$, are relatively weakly compact.

Remark 3.3 For $G \in \mathcal{G}$, properties (i) and (iii) in Definition 2.3 give that joint lower semicontinuity of $G$ is equivalent to continuity of $G(\mathbb{Q}, \cdot), \mathbb{Q} \in \mathcal{M}_{1}(\mathbb{P})$. Preorders satisfying certain additional continuity axioms admit the representation (2.3) for such $G \in \mathcal{G}$; the corresponding utility functional is then continuous, see [11, Theorem 7]. For concave utility functionals, continuity from below implies weak compactness of the level sets; see [30, Lemma 4.22]. As for homothetic preferences, any $G \in \mathcal{G}$ of the form $G(\mathbb{Q}, t)=g(\mathbb{Q}) h(t)$, with $h: \mathbb{R} \rightarrow \mathbb{R}$ continuous and $g: \mathcal{M}_{1}(\mathbb{P}) \rightarrow \mathbb{R}$ assigning finite penalty only to measures within some compact set, satisfies Assumption 3.2.

${ }^{11}$ See e.g. [44], where it is shown that the classical utility maximization problem admits a solution if and only if the market satisfies the no-arbitrage condition $\mathrm{NA}_{1}$. 
Note also that weak lower semicontinuity of $G$ implies that $\mathcal{Q}_{t}(c)$ is weakly closed, and thus Assumption 3.2 yields in fact weak compactness of $\mathcal{Q}_{t}(c)$. For multiplepriors preferences, certain minimax results were established without compactness assumptions in [4].

To define the dual problem, let $\mathcal{Y}(y)$ be the set of all positive $\mathbb{P}$-supermartingales such that $Y_{0}=y$ and $X Y$ is a $\mathbb{P}$-supermartingale for all $X \in \mathcal{X}(1)$. Further, let $V: \mathbb{R}_{+} \rightarrow \mathbb{R}$ be given by

$$
V(y)=\sup _{x>0}(U(x)-x y), \quad y>0 .
$$

Identifying each measure $\mathbb{Q} \in \mathcal{Q}$ with its Radon-Nikodým derivative $Z^{\mathbb{Q}}=\frac{\mathrm{d} \mathbb{Q}}{\mathrm{d}}$, and employing the convention that $Z V(Y / Z):=0$ on $\{Z=0\}, Z, Y \in L_{+}^{0}$, we define the dual value function $v(\cdot ; x), x>0$, by

$$
v(y ; x):=\inf _{\mathbb{Q} \in \mathcal{Q}} \inf _{Y \in \mathcal{Y}(y)} G\left(\mathbb{Q}, x y+\mathrm{E}\left[Z^{\mathbb{Q}} V\left(\frac{Y_{T}}{Z^{\mathbb{Q}}}\right)\right]\right), \quad y>0 .
$$

We first show that the dual problem admits a solution.

Proposition 3.4 Let $G \in \mathcal{G}$ and $U \in \mathcal{U}$ be given and suppose that Assumptions 2.8 and 3.2 hold. If $v(y ; x)<\infty$, then the infimum in (3.1) is attained. Moreover, there exists a solution $(\widehat{\mathbb{Q}}, \widehat{Y})$ that is maximal in the sense that any other solution $(\mathbb{Q}, Y)$, satisfies $\mathbb{Q} \ll \widehat{\mathbb{Q}}$ and $Y_{T} / Z^{\mathbb{Q}}=\widehat{Y}_{T} / Z^{\widehat{\mathbb{Q}}} \mathbb{Q}$-a.s.

The next result relates the primal and dual value functions; for a discussion of the weaker sufficient conditions in the variational case, see Remark 4.7 below.

Theorem 3.5 Let $G \in \mathcal{G}$ and $U \in \mathcal{U}$ be given and suppose that Assumptions 2.8 and 3.2 hold and that (2.9) holds for all $\mathbb{Q} \in \mathcal{Q}$. Then we have the relations

$$
u(x)=\inf _{\mathbb{Q} \in \mathcal{Q}} \sup _{X \in \mathcal{X}(x)} G\left(\mathbb{Q}, \mathrm{E}^{\mathbb{Q}}\left[U\left(X_{T}\right)\right]\right)
$$

and

$$
u(x)=\inf _{y>0} v(y ; x) .
$$

The study of the dual problem is in fact not needed to obtain existence of a solution to the primal problem. Indeed, the proof of Theorem 3.1 relies on properties of the auxiliary problem (2.8) and thus rather on the study of the dual version thereof (cf. (4.1) below). Nevertheless, the dual counterpart is of interest, for it gives a further understanding of the optimal strategy; see Sect. 3.3. Moreover, the dual problem is more tractable. This is in fact particularly evident for ambiguity-averse preferences where the dual problem amounts to the search for a pure infimum, whereas the primal problem features a saddle structure. Notably, most articles providing explicit solutions for specific utility and penalty functions consider the dual rather than the 
primal problem. One would therefore expect the dual formulation to be helpful in obtaining explicit results also for quasiconcave preferences.

\subsection{The relation between the primal and dual solution}

The next result establishes the relation between the primal and dual solution and the existence of a saddle point. ${ }^{12}$ This is of particular interest since it yields the existence of an equivalent auxiliary problem and results on the uniqueness of the optimal strategy.

Theorem 3.6 Let $G \in \mathcal{G}$ and $U \in \mathcal{U}$ be given and suppose that the assumptions of Theorem 3.5 hold. Then the primal problem admits a saddle point if and only if the infimum in (3.3) is attained for some $y^{*}>0$. Specifically, for any solution $(\widehat{\mathbb{Q}}, \widehat{Y})$ to the dual problem at the level $y^{*}$ and any primal solution $\bar{X}_{T}$, the pair $\left(\bar{X}_{T}, \widehat{\mathbb{Q}}\right)$ is a saddle point for the primal problem. If in addition $G(\mathbb{Q}, \cdot)$ is strictly increasing, then

$$
\bar{X}_{T}=-V^{\prime}\left(\frac{\widehat{Y}_{T}}{Z^{\widehat{\mathbb{Q}}}}\right) \quad \widehat{\mathbb{Q}} \text {-a.s. }
$$

We note that a saddle point always exists for the variational case; see [71]. We also have the following result; notably, much of the literature on ambiguity-averse portfolio optimization focuses on positive utility functions, see among others $[37,68]$.

Proposition 3.7 Let $G \in \mathcal{G}$ and $U \in \mathcal{U}$ be given and suppose that the assumptions of Theorem 3.5 hold. Suppose further that $U: \mathbb{R}_{+} \rightarrow \mathbb{R}_{+}$. Then the primal problem admits a saddle point.

We now discuss the implications of the above results. To this end, let $\widehat{\mathbb{Q}}$ be the first component in the maximal solution to the dual problem at level $y^{*}>0$. Relation (3.4) then implies that the solution $\bar{X}_{T}$ is $\widehat{\mathbb{Q}}$-a.s. unique. Consequently, if $\widehat{\mathbb{Q}} \sim \mathbb{P}$, the solution is $\mathbb{P}$-a.s. unique and can be recovered from the dual solution. In general, $\widehat{\mathbb{Q}}$ need not be equivalent to $\mathbb{P}$; nevertheless, an optimal strategy can still be constructed from a given dual solution by superreplication of an appropriate claim (see Corollary 2.6 in [71]). The existence of a saddle point also implies that there is an auxiliary investment problem producing the same optimal behaviour as the original criterion: the auxiliary problem $(2.8)$ defined with respect to $\widehat{\mathbb{Q}}$ admits a solution which $\widehat{\mathbb{Q}}$-a.s. coincides with the solution to the original problem. In particular, the uniqueness $\widehat{\mathbb{Q}}$-a.s. of the optimal strategy is thus natural since this equivalent problem has a concave objective. Since the measure $\widehat{\mathbb{Q}}$ is part of the solution to the original problem, it is not a priori known. In particular, it depends on both $G$ and $U$. Nevertheless, the fact that the investor's behaviour is equivalent to that of an investor with market views

${ }^{12}$ We say that $\left(\bar{X}_{T}, \overline{\mathbb{Q}}\right)$, with $\bar{X} \in \mathcal{X}(x)$ and $\overline{\mathbb{Q}} \in \mathcal{Q}$, is a saddle point if

$$
u(x)=\sup _{X \in \mathcal{X}(x)} G\left(\overline{\mathbb{Q}}, \mathrm{E}^{\overline{\mathbb{Q}}}\left[U\left(X_{T}\right)\right]\right)=\inf _{\mathbb{Q} \in \mathcal{Q}} G\left(\mathbb{Q}, \mathrm{E}^{\mathbb{Q}}\left[U\left(\bar{X}_{T}\right)\right]\right)=G\left(\overline{\mathbb{Q}}, \mathrm{E}^{\overline{\mathbb{Q}}}\left[U\left(\bar{X}_{T}\right)\right]\right) .
$$


specified by $\widehat{\mathbb{Q}}$ is informative. For example, it implies that the market equilibrium formed by ambiguity-averse investors coincides with that formed by expected utility maximizers with different market views; we refer to [55] for a study of the effects of diverging market views on the endogenously defined price process. For preferences only satisfying the weaker properties of Theorem 3.1, there need not exist an auxiliary investment problem. The question to which extent the optimal strategy is then unique is left for future study.

We conclude with some remarks on the (lack of) time consistency. For general $G \in \mathcal{G}$, Problem 2.6 is not a time-consistent investment problem. This is in line with Remark 3.5 in [71], where it was argued that for investment under model uncertainty, time consistency is not always a natural prerequisite. In effect, however, the optimal strategy is only optimal in a precommitment sense, which leads to a number of interesting questions. First, there are results providing tractable solutions to multi-period and continuous-time formulations of the (time-inconsistent) mean-variance criterion (see, among others, [7, 17]). It would be interesting to see whether these methods could be applied to more general quasiconcave preferences. Second, one may further study the subclass of time-consistent quasiconcave preferences. While time consistency is of interest in its own right, it also enables the use of stochastic control methods and is necessary for extending to the quasiconcave case the explicit results obtained in terms of PDEs and BSDEs for variational preferences. Time consistency of quasiconcave utility functionals remains, however, an open problem and feasible explicit examples are few. Indeed, while necessary and sufficient conditions for temporal consistency of convex risk measures were established in [20], see also e.g. [6, 28 ], such results are yet lacking for the quasiconvex case. ${ }^{13}$ Finally, recall that the risk preferences of the investor are modelled via a standard continuous and concave utility function in (2.7). While this is a natural assumption, the value function $u(x)$ satisfies only weaker properties. Hence, an application of dynamic programming arguments would also require a study of Problem 2.6 under weaker assumptions on the utility function.

\section{Proofs}

The results herein extend results established for variational preferences in [71] (see $[68,72]$ for the coherent case). Our proofs are therefore inspired by those in the former articles. Specifically, also here the idea is to establish results for the risk- and ambiguity-averse problem by relying on results for the auxiliary problem (2.8). The differences induced by the more general criteria we consider are mainly of two types. First, we need throughout to work with quasiconvexity and semicontinuity, as opposed to convexity and continuity, which requires a more elaborate analysis. Second, the present case calls for a more detailed study of the auxiliary problem. For $\mathbb{Q} \sim \mathbb{P}$, this is the classical utility maximization problem studied in $[50,51]$; for $\mathbb{Q} \ll \mathbb{P}$, this is no longer the case. In [71], this is dealt with by use of certain limiting arguments

\footnotetext{
${ }^{13}$ However, we note that $[32,33]$, see also [5], initiated such a programme via the study of conditional quasiconvex risk measures, and that questions of temporal consistency and its relation to $g$-expectations were addressed within the framework of nonlinear expectations in [66].
} 
which imply that only equivalent measures need to be taken into consideration. This approach is closely related to the following property of a monetary utility functional: if there exists an equivalent measure for which the penalty is finite, then the set of absolutely continuous measures appearing in the robust representation can be replaced by the equivalent ones; see [48]. As it turns out, convexity of $G(\cdot, t), t \in \mathbb{R}$, is sufficient for this property to hold, and for this case we may proceed along similar lines. For our general case, the weaker properties of $G$ imply, however, that this approach does not apply. We therefore proceed by first establishing the relevant properties for a general auxiliary problem. In particular, we thus obtain alternative proofs of some of the duality results in [71]; see Remark 4.7.

The rest of this section is organized as follows. We first establish the properties of the auxiliary investment problem in Sect. 4.1. In Sect. 4.2, we prove the existence of an optimizer and the upper semicontinuity of the value function. In Sect. 4.3, we proceed with the various duality results.

Throughout, we identify each measure $\mathbb{Q} \in \mathcal{Q}$ with its Radon-Nikodým derivative $Z=\frac{\mathrm{d} \mathbb{Q}}{\mathrm{dP}}$, and without further notice we index functions by $Z$ rather than $\mathbb{Q}$. Further, for $V: \mathbb{R}_{+} \rightarrow \mathbb{R}$ given in Sect. 3.2, we employ the convention:

$$
Z V(Y / Z):=0 \text { on }\{Z=0\}, \quad \text { for } Z, Y \in L_{+}^{0} .
$$

\subsection{The auxiliary investment problem and its significance}

We first introduce the dual auxiliary value function by

$$
v_{\mathbb{Q}}(y):=\inf _{Y \in \mathcal{Y}(y)} \mathrm{E}\left[Z V\left(Y_{T} / Z\right)\right], \quad y>0, \mathbb{Q} \in \mathcal{M}_{1}(\mathbb{P}),
$$

where $\mathcal{Y}(y)$ is as defined in Sect. 3.2. The dual value function introduced in (3.1) then admits the representation $v(y ; x)=\inf \left\{G\left(\mathbb{Q}, v_{\mathbb{Q}}(y)+x y\right): \mathbb{Q} \in \mathcal{Q}\right\}$.

Remark 4.1 The dual value function also admits the alternative representation

$$
v_{\mathbb{Q}}(y)=\inf _{Y \in \mathcal{Y}_{\mathbb{Q}}(y)} \mathrm{E}^{\mathbb{Q}}\left[V\left(Y_{T}\right)\right],
$$

where $\mathcal{Y}_{\mathbb{Q}}(y)$ denotes the set of all positive $\mathbb{Q}$-supermartingales such that $Y_{0}=y$ and $X Y$ is a $\mathbb{Q}$-supermartingale for all $X \in \mathcal{X}(1)$; see [71, Lemma 4.2].

For $\mathbb{Q} \sim \mathbb{P}$, the auxiliary investment problem (2.8) is the classical utility maximization problem which was solved in full generality in $[50,51]$. We recall that their results require the market to be arbitrage-free in the sense that the set of local martingale measures is non-empty, $\mathcal{M} \neq \emptyset$, and that this assumption is used to establish the duality between the primal and dual optimization objects. Specifically, the sets $\mathcal{X}(x)$ and $\mathcal{Y}(y)$ in the primal and dual problems may, respectively, be replaced by the sets $\mathcal{C}(x)$ and $\mathcal{D}(y)$, where the latter are given by

$$
\mathcal{C}(x):=\left\{g \in L_{+}^{0}: g \leq X_{T} \mathbb{P} \text {-a.s. for some } X \in \mathcal{X}(x)\right\}
$$


and

$$
\mathcal{D}(y):=\left\{h \in L_{+}^{0}: h \leq Y_{T} \mathbb{P} \text {-a.s. for some } Y \in \mathcal{Y}(y)\right\} .
$$

Under the assumption that $\mathcal{M} \neq \emptyset$, the following assertion was then proved in [50]:

$$
\begin{aligned}
& g \in \mathcal{C}(x) \Longleftrightarrow g \geq 0 \text { and } \sup _{h \in \mathcal{D}(y)} \mathrm{E}[g h] \leq x y, \\
& h \in \mathcal{D}(y) \Longleftrightarrow h \geq 0 \text { and } \sup _{g \in \mathcal{C}(x)} \mathrm{E}[g h] \leq x y .
\end{aligned}
$$

For $\mathbb{Q} \ll \mathbb{P}$, the auxiliary problem differs from the classical one; while the objective function uses the market measure $\mathbb{Q}$, the set of admissible trading strategies are still required to be feasible $\mathbb{P}$-a.s., and it is also not clear in what sense the market model is arbitrage-free under $\mathbb{Q}$. Crucially, however, by viewing the auxiliary problem as a classical utility maximization problem under the measure $\mathbb{P}$, with respect to the stochastic utility function $Z U(\cdot)$ where $Z$ is the associated Radon-Nikodým derivative, it turns out that the problem may be addressed in a similar manner as the classical one. We emphasize that the following result follows by minor modifications of the proofs in $[50,51]$ (see also [27, 62]); we demonstrate the altered steps in the Appendix.

Lemma 4.2 Let $U: \mathbb{R}_{+} \rightarrow \mathbb{R}$ be strictly increasing, strictly concave and satisfy the Inada conditions (2.6). Suppose that $u_{Z}\left(x_{0}\right)<\infty$ for some $x_{0}>0$. Then the functions $u_{Z}(x)$ and $v_{Z}(y)$ satisfy the relations

$$
v_{Z}(y)=\sup _{x>0}\left(u_{Z}(x)-x y\right) \quad \text { and } u_{Z}(y)=\inf _{y>0}\left(v_{Z}(x)+x y\right) \text {, }
$$

and

$$
u_{Z}^{\prime}(0)=\infty \quad \text { and } \quad v_{Z}^{\prime}(\infty)=0
$$

Under the additional assumption that $A E(U)<1$, it also holds that

$$
u_{Z}^{\prime}(\infty)=0 \quad \text { and } \quad v_{Z}^{\prime}(0)=-\infty
$$

the set $\left\{Z U^{+}(g): g \in \mathcal{C}(x)\right\}$ is $\mathbb{P}$-uniformly integrable, and the primal problem admits a solution.

For $\mathbb{Q} \sim \mathbb{P}$, it was proved in [51] that for utility functions which do not necessarily satisfy $A E(U)<1$, a necessary and sufficient condition for Lemma 4.2 to hold is that $v_{\mathbb{Q}}(y)<\infty, y>0$. This is true also for $\mathbb{Q} \ll \mathbb{P}$. In consequence, the results herein hold without the asymptotic elasticity condition if we impose directly the assumption that $v_{\mathbb{Q}}<\infty$, for all $\mathbb{Q} \in \mathcal{Q}$.

\subsection{Proof of the existence of an optimal investment strategy}

We first provide the following proof: 
Proof of Lemma 2.9. Note that the finiteness is immediate. Next, take $x \leq y$ and let $X \in \mathcal{X}(x)$. Then $X \leq X+y-x \in \mathcal{X}(y)$. Since $\inf _{\mathbb{Q} \in \mathcal{Q}} G(\mathbb{Q}, \cdot)$ is nondecreasing, it follows that

$$
\inf _{\mathbb{Q} \in \mathcal{Q}} G\left(\mathbb{Q}, \mathrm{E}^{\mathbb{Q}}[U(X)]\right) \leq \inf _{\mathbb{Q} \in \mathcal{Q}} G\left(\mathbb{Q}, \mathrm{E}^{\mathbb{Q}}[U(X+y-x)]\right) \leq u(y) .
$$

Since $X \in \mathcal{X}(x)$ was chosen arbitrarily, we obtain $u(x) \leq u(y)$. Finally, for some $\lambda \in[0,1]$, let $z:=\lambda x+(1-\lambda) y$ and take $X^{x} \in \mathcal{X}(x)$ and $X^{y} \in \mathcal{X}(y)$. Then we obtain that $\bar{X}:=\lambda X^{x}+(1-\lambda) X^{y} \in \mathcal{X}(z)$. Since $U$ is concave and $\inf _{\mathbb{Q} \in \mathcal{Q}} G(\mathbb{Q}, \cdot)$ is nondecreasing, it follows that

$$
u(z) \geq \min \left\{\inf _{\mathbb{Q} \in \mathcal{Q}} G\left(\mathbb{Q}, \mathrm{E}^{\mathbb{Q}}\left[U\left(X^{x}\right)\right]\right), \inf _{\mathbb{Q} \in \mathcal{Q}} G\left(\mathbb{Q}, \mathrm{E}^{\mathbb{Q}}\left[U\left(X^{y}\right)\right]\right)\right\} .
$$

Taking the supremum over $X^{x} \in \mathcal{X}(x)$ and $X^{y} \in \mathcal{X}(y)$ gives $u(z) \geq \min \{u(x), u(y)\}$.

Next, we define the function $\Phi: L_{+}^{0} \rightarrow \mathbb{R}$ by

$$
\Phi(g):=\inf _{\mathbb{Q} \in \mathcal{Q}} G\left(\mathbb{Q}, \mathrm{E}^{\mathbb{Q}}[U(g)]\right) .
$$

The existence of an optimizer is now proved by first establishing upper semicontinuity and quasiconcavity of this objective function. Similarly to the classical case, the result can then be argued using a Komlós-type result.

Lemma 4.3 Let $G \in \mathcal{G}$ and $U \in \mathcal{U}$ be given and suppose that Assumption 2.8 holds, that $G(\cdot, t), t \in \mathbb{R}$, is convex, that $G$ is finite on $\mathcal{Q} \times \mathbb{R}$, and that (2.9) holds for some $\mathbb{Q} \in \mathcal{Q}_{e}$ with $\mathcal{Q}_{e}:=\{\mathbb{Q} \in \mathcal{Q}: \mathbb{Q} \sim \mathbb{P}\}$. Then

$$
\Phi(g)=\inf _{\mathbb{Q} \in \mathcal{Q}_{e}} G\left(\mathbb{Q}, \mathrm{E}^{\mathbb{Q}}[U(g)]\right) \quad \text { for } g \in \mathcal{C}(x) .
$$

Proof Let $g \in \mathcal{C}(x)$ be fixed. Since $G$ has an asymptotic maximum in the sense of Definition 2.3 and there exists some $\mathbb{Q} \in \mathcal{Q}$ for which $u_{\mathbb{Q}}(x)<\infty$, the set $\mathcal{Q}$ in (4.6) may without loss of generality be replaced by

$$
\mathcal{Q}(g)=\left\{\mathbb{Q} \in \mathcal{Q}: \mathrm{E}^{\mathbb{Q}}[U(g)]<\infty\right\} .
$$

For $\mathbb{Q}_{0} \in \mathcal{Q}(g) \backslash \mathcal{Q}_{e}$, take $\mathbb{Q}_{1} \in \mathcal{Q}_{e}$ such that (2.9) holds and define for $t \in[0,1]$ the measure $\mathbb{Q}_{t}$ via the Radon-Nikodým derivative $Z_{t}:=(1-t) Z_{0}+t Z_{1}$, where $Z_{0}$ and $Z_{1}$ correspond, respectively, to $\mathbb{Q}_{0}$ and $\mathbb{Q}_{1}$. Then $\mathbb{Q}_{t} \in \mathcal{Q}_{e}$ for $t \in(0,1]$. Note that $t \mapsto \mathrm{E}\left[Z_{t} U(g)\right]$ is affine and finite, and thus continuous for $t \in[0,1]$. Further, $t \mapsto G\left(Z_{t}, s\right)$ is convex and therefore continuous since it is finite for $(t, s) \in[0,1] \times \mathbb{R}$. Using that $G\left(Z_{t}, \cdot\right)$ is nondecreasing as well as upper semicontinuous, we thus obtain that $(t, s) \mapsto G\left(Z_{t}, s\right)$ is jointly upper semicontinuous. In consequence,

$$
t \mapsto G\left(Z_{t}, \mathrm{E}\left[Z_{t} U(g)\right]\right)
$$


is upper semicontinuous, and it follows that

$$
G\left(Z_{0}, \mathrm{E}\left[Z_{0} U(g)\right]\right) \geq \limsup _{t \rightarrow 0} G\left(Z_{t}, \mathrm{E}\left[Z_{t} U(g)\right]\right) .
$$

The minimization over $\mathcal{Q}(g)$ in (4.6) can therefore be further replaced by minimization over $\mathcal{Q}_{e}$, and we conclude.

Lemma 4.4 Let $G \in \mathcal{G}$ and $U \in \mathcal{U}$ and suppose that the assumptions of Theorem 3.1 hold. Then the function $\Phi$ given in (4.6) is quasiconcave and upper semicontinuous with respect to convergence in probability on $\mathcal{C}(x)$.

Proof Suppose first that $u_{\mathbb{Q}}(x)<\infty$ for all $\mathbb{Q} \in \mathcal{Q}$. Let $\left(g_{n}\right) \subseteq \mathcal{C}(x)$ be a sequence converging in probability to some $g \in \mathcal{C}(x)$. According to Lemma 4.2, the set $\left\{U^{+}\left(g_{n}\right): n \in \mathbb{N}\right\}$ is then $\mathbb{Q}$-uniformly integrable, for $\mathbb{Q} \in \mathcal{Q}$. On the other hand, an application of Fatou's lemma (to a suitable subsequence) gives that $\limsup _{n \rightarrow \infty} \mathrm{E}^{\mathbb{Q}}\left[U^{-}\left(g_{n}\right)\right] \leq \mathrm{E}^{\mathbb{Q}}\left[U^{-}(g)\right]$. Hence, for each $\mathbb{Q} \in \mathcal{Q}$, the mapping $g \mapsto \mathrm{E}^{\mathbb{Q}}[U(g)]$ is upper semicontinuous for convergence in probability on $\mathcal{C}(x)$. Recalling that $g \mapsto \mathrm{E}^{\mathbb{Q}}[U(g)]$ is also concave, and that $G(\mathbb{Q}, \cdot)$ is right-continuous and nondecreasing, it follows that for each $\mathbb{Q} \in \mathcal{Q}$, the mapping $g \mapsto G\left(\mathbb{Q}, \mathrm{E}^{\mathbb{Q}}[U(g)]\right)$ is also quasiconcave and upper semicontinuous on $\mathcal{C}(x)$. In turn, since

$$
\{g \in \mathcal{C}(x): \Phi(g) \geq m\}=\bigcap_{\mathbb{Q} \in \mathcal{Q}}\left\{g \in \mathcal{C}(x): G\left(\mathbb{Q}, \mathrm{E}^{\mathbb{Q}}[U(g)]\right) \geq m\right\}
$$

and quasiconcavity and upper semicontinuity are characterized by convexity and closedness of the upper level sets, these properties are preserved also by the function $\Phi$.

When $G(\cdot, t)$ is convex, the result follows by use of the same arguments, applying then Lemma 4.2 only to measures $\mathbb{Q} \in \mathcal{Q}_{e}$ by use of Lemma 4.3.

Proof of Theorem 3.1 First note that we may without loss of generality replace the set $\mathcal{X}(x)$ in (2.7) by the set $\mathcal{C}(x)$ defined above (4.2) and consider the associated problem. Indeed,

$$
u(x)=\sup _{g \in \mathcal{C}(x)} \Phi(g),
$$

and if (4.7) is attained for some $g \in \mathcal{C}(x)$, then there exists $X \in \mathcal{X}(x)$ such that $X_{T} \geq g$, and thus that $X$ solves the original problem.

Let $\left(g_{n}\right) \subseteq \mathcal{C}(x)$ be a sequence such that $\Phi\left(g_{n}\right) \nearrow u(x)$. Since $g_{n} \geq 0, n \in \mathbb{N}$, there exists a further sequence $\tilde{g}_{n} \in \operatorname{conv}\left(g_{n}, g_{n+1}, \ldots\right)$ converging $\mathbb{P}$-a.s. to some $g$; see e.g. [21, Lemma A1.1]. Since $\mathcal{C}(x)$ is convex, $\left(\tilde{g}_{n}\right) \subseteq \mathcal{C}(x)$. By Fatou's lemma, it then follows from (4.2) that $g \in \mathcal{C}(x)$. Due to the quasiconcavity of $\Phi$ and the fact that $\Phi\left(g_{n}\right)$ increases to $u(x)$, we have that

$$
\Phi\left(\tilde{g}_{n}\right) \geq \inf _{k \geq n} \Phi\left(g_{k}\right)=\Phi\left(g_{n}\right) .
$$

In consequence, also $\left(\tilde{g}_{n}\right)$ is an optimizing sequence. The optimality of $g$ now follows from the upper semicontinuity via $\Phi(g) \geq \limsup _{n \rightarrow \infty} \Phi\left(\tilde{g}_{n}\right)=u(x)$. 
It remains to argue the upper semicontinuity of $u$. To this end, recall from Lemma 2.9 that $u$ is nondecreasing and finite. Let $x_{0} \in \mathbb{R}_{+}$and $x_{n}=x_{0}+\frac{1}{n}, n \in \mathbb{N}$. Then

$$
\lim _{n \rightarrow \infty} u\left(x_{n}\right)=\limsup _{x \downarrow x_{0}} u(x) .
$$

For each $n \in \mathbb{N}$, let $g_{n} \in \mathcal{C}\left(x_{n}\right)$ be such that $u\left(x_{n}\right)=\Phi\left(g_{n}\right)$. Applying once again Lemma A1.1 in [21], we obtain a sequence $\tilde{g}_{n} \in \operatorname{conv}\left(g_{n}, g_{n+1}, \ldots\right)$ such that $\tilde{g}_{n} \rightarrow g \mathbb{P}$-a.s. for some $g$. Due to the quasiconcavity of $\Phi$ and the fact that $\Phi\left(g_{n}\right)$ is decreasing in $n$, we have that

$$
\Phi\left(\tilde{g}_{n}\right) \geq \inf _{i=n, n+1, \ldots} \Phi\left(g_{i}\right)=\lim _{n \rightarrow \infty} u\left(x_{n}\right) .
$$

Further, note that $g_{n}, g_{n+1}, \ldots \in \mathcal{C}\left(x_{n}\right)$. Since $\mathcal{C}\left(x_{n}\right)$ is convex, it follows that $\tilde{g}_{n} \in \mathcal{C}\left(x_{n}\right), n \in \mathbb{N}$. Hence, according to (4.2), $g \in \mathcal{C}\left(x_{0}\right)$. Moreover, for any $n \in \mathbb{N}$, we have $g \in \mathcal{C}\left(x_{n}\right)$ and $\tilde{g}_{n}, \tilde{g}_{n+1}, \ldots \in \mathcal{C}\left(x_{n}\right)$; use of the upper semicontinuity of $\Phi$ then yields

$$
\Phi(g) \geq \limsup _{n \rightarrow \infty} \Phi\left(\tilde{g}_{n}\right) \geq \lim _{n \rightarrow \infty} u\left(x_{n}\right),
$$

which allows us to conclude.

\subsection{Proof of the duality results}

In preparation for the proof of Theorem 3.5 , note that since $G(Z, \cdot)$ is nondecreasing, it follows immediately that

$$
u(x) \leq \inf _{\mathbb{Q} \in \mathcal{Q}} G\left(\mathbb{Q}, u_{\mathbb{Q}}(x)\right) \leq \inf _{\mathbb{Q} \in \mathcal{Q}} G\left(\mathbb{Q}, \inf _{y>0}\left(v_{\mathbb{Q}}(y)+x y\right)\right)=\inf _{y>0} v(y ; x) .
$$

We argue that the inequalities hold as equalities, using, respectively, the minimax theorem by Sion [74] and Lemma 4.2. To this end, we first establish two lemmas.

Lemma 4.5 Let $G \in \mathcal{G}$ and $U \in \mathcal{U}$ be given and suppose that the assumptions of Theorem 3.5 hold. Then for any $\varepsilon>0$,

$$
\sup _{g \in \mathcal{C}(x)} \inf _{Z \in \mathcal{Q}} G(Z, \mathrm{E}[Z U(\varepsilon+g)])=\inf _{Z \in \mathcal{Q}} \sup _{g \in \mathcal{C}(x)} G(Z, \mathrm{E}[Z U(\varepsilon+g)]) .
$$

Proof Let $\Psi_{\varepsilon}(Z, g):=G(Z, \mathrm{E}[Z U(\varepsilon+g)])$ for $Z \in \mathcal{Q}, g \in \mathcal{C}(x)$, and note that for each $g \in \mathcal{C}(x)$,

$$
\inf _{Z \in \mathcal{Q}} \Psi_{\varepsilon}(Z, g) \leq \sup _{g \in \mathcal{C}(x)} \inf _{Z \in \mathcal{Q}} \Psi_{\varepsilon}(Z, g) \leq u(x+\varepsilon)
$$

According to Lemma 2.9, $u(x)<\infty, x>0$. In consequence, introducing the set $\mathcal{Q}^{g}:=\left\{Z \in \mathcal{Q}: \Psi_{\varepsilon}(Z, g) \leq c\right\}$ with $c=u(x+\varepsilon) \vee 0+1$, it follows that $\inf _{Z \in \mathcal{Q}} \Psi_{\varepsilon}(Z, g)=\inf _{Z \in \mathcal{Q}^{g}} \Psi_{\varepsilon}(Z, g)$. Further, since $\mathrm{E}[Z U(\varepsilon+g)] \geq U(\varepsilon) \wedge 0$ for 
$Z \in \mathcal{Q}$, it holds for any $Z \in \mathcal{Q}^{g}$ that $G(Z, t) \leq c$ with $t:=U(\varepsilon) \wedge 0$; that is, for all $g \in \mathcal{C}(x), \mathcal{Q}^{g} \subseteq \mathcal{Q}_{t}(c)$. Hence we obtain

$$
\sup _{g \in \mathcal{C}(x)} \inf _{Z \in \mathcal{Q}} \Psi_{\varepsilon}(Z, g)=\sup _{g \in \mathcal{C}(x)} \inf _{Z \in \mathcal{Q}_{t}(c)} \Psi_{\varepsilon}(Z, g) .
$$

Next, since $U(\varepsilon+\cdot)$ is bounded from below, we may apply Fatou's lemma to obtain lower semicontinuity, with respect to convergence in probability, of the mapping $Z \mapsto \mathrm{E}[Z U(\varepsilon+g)], g \in \mathcal{C}(x)$. Since $\mathcal{Q}_{t}(c)$ is uniformly integrable according to Assumption 3.2 , this is equivalent to lower semicontinuity with respect to convergence in $L^{1}$. Since the mapping is affine, it is therefore weakly lower semicontinuous on $\mathcal{Q}_{t}(c)$. Recalling that $G$ is quasiconvex and jointly lower semicontinuous, we thus obtain that the mapping

$$
Z \mapsto \Psi_{\varepsilon}(Z, g), \quad g \in \mathcal{C}(x),
$$

is quasiconvex and weakly lower semicontinuous on $\mathcal{Q}_{t}(c)$. On the other hand, as established in the proof of Lemma 4.4, the mapping

$$
g \mapsto \Psi_{\varepsilon}(Z, g), \quad Z \in \mathcal{Q},
$$

is quasiconcave and upper semicontinuous for convergence in probability on $\mathcal{C}(x)$. Indeed, since $U$ is concave, the fact that $\left\{U^{+}(g): g \in \mathcal{C}(x)\right\}$ is uniformly integrable implies that so is $\left\{U^{+}(\varepsilon+g): g \in \mathcal{C}(x)\right\}$, and the same argument goes through.

Recall that $\mathcal{C}(x)$ is convex. Moreover, for $Z, \bar{Z} \in \mathcal{Q}_{t}(c)$,

$$
G(\lambda Z+(1-\lambda) \bar{Z}, t) \leq \max \{G(Z, t), G(\bar{Z}, t)\} \leq c .
$$

Hence, also $\mathcal{Q}_{t}(c)$ is convex. It is also weakly compact; cf. Remark 3.3.

The assumptions of Theorem 3.4 in Sion [74] are thus satisfied, and we may apply this result (specifically Corollary 3.3 therein) to obtain

$$
\sup _{g \in \mathcal{C}(x)} \inf _{Z \in \mathcal{Q}_{t}(c)} \Psi_{\varepsilon}(Z, g)=\inf _{Z \in \mathcal{Q}_{t}(c)} \sup _{g \in \mathcal{C}(x)} \Psi_{\varepsilon}(Z, g) .
$$

Combining (4.8) and (4.10), we have

$$
\inf _{Z \in \mathcal{Q}} \sup _{g \in \mathcal{C}(x)} \Psi_{\varepsilon}(Z, g) \leq \sup _{g \in \mathcal{C}(x)} \inf _{Z \in \mathcal{Q}} \Psi_{\varepsilon}(Z, g),
$$

and since the reverse inequality is immediate, this allows us to conclude.

Lemma 4.6 Let $G \in \mathcal{G}$ and $U \in \mathcal{U}$ be given and suppose that Assumption 2.8 holds. Then

$$
\inf _{Z \in \mathcal{Q}} \sup _{g \in \mathcal{C}(x)} G(Z, \mathrm{E}[Z U(g)])=\inf _{y>0} v(y ; x)
$$

Proof According to Assumption 2.8, $\mathcal{Q}^{f} \neq \emptyset$ with $\mathcal{Q}^{f}=\left\{\mathbb{Q} \in \mathcal{Q}: u_{\mathbb{Q}}(x)<\infty, x>0\right\}$. Combining this with the fact that $G$ is nondecreasing in its second argument and 
has an asymptotic maximum in the sense of Definition 2.3, and using the conjugacy relation (4.3) between $u_{\mathbb{Q}}(x)$ and $v_{\mathbb{Q}}(y)$ for $\mathbb{Q} \in \mathcal{Q}^{f}$, we obtain

$$
\begin{aligned}
\inf _{Z \in \mathcal{Q}} G\left(Z, u_{Z}(x)\right) & =\inf _{Z \in \mathcal{Q}^{f}} G\left(Z, u_{Z}(x)\right) \\
& =\inf _{Z \in \mathcal{Q}^{f}} G\left(Z, \inf _{y>0}\left(v_{Z}(y)+x y\right)\right) \\
& =\inf _{y>0} \inf _{Z \in \mathcal{Q}^{f}} G\left(Z, v_{Z}(y)+x y\right) .
\end{aligned}
$$

Recall that $v(y ; x)=\inf \left\{G\left(Z, v_{Z}(y)+x y\right): Z \in \mathcal{Q}\right\}$. It therefore only remains to argue that

$$
\inf _{Z \in \mathcal{Q}^{f}} G\left(Z, v_{Z}(y)+x y\right)=\inf _{Z \in \mathcal{Q}} G\left(Z, v_{Z}(y)+x y\right), \quad y>0 .
$$

The inequality " $\geq$ " is immediate since $\mathcal{Q}^{f} \subseteq \mathcal{Q}$. Hence, for $y>0$ fixed, using again that $G$ is nondecreasing in its second argument and has an asymptotic maximum, we note that without loss of generality, $\tilde{\mathcal{Q}}:=\left\{Z \in \mathcal{Q}: v_{Z}(y)<\infty\right\} \neq \emptyset$ and the set $\mathcal{Q}$ on the right-hand side of (4.11) can then be replaced by $\tilde{\mathcal{Q}}$. On the other hand, $v_{Z}(y)<\infty$ implies that $u_{Z}(x)<\infty, x>0$ (cf. Lemma 3.5 in [72] along with the proof of Lemma 4.2). Hence, $\tilde{\mathcal{Q}} \subseteq \mathcal{Q}^{f}$ which yields the reverse inequality.

Proof of Theorem 3.5 Applying Lemma 4.5, we obtain

$$
\begin{aligned}
u(x) & \leq \inf _{Z \in \mathcal{Q}} \sup _{g \in \mathcal{C}(x)} G(Z, \mathrm{E}[Z U(g)]) \\
& \leq \sup _{g \in \mathcal{C}(x)} \inf _{Z \in \mathcal{Q}} G(Z, \mathrm{E}[Z U(\varepsilon+g)]) \leq u(x+\varepsilon) .
\end{aligned}
$$

Recalling from Theorem 3.1 that $u(x)$ is upper semicontinuous, sending $\varepsilon$ to zero, we obtain

$$
u(x)=\inf _{Z \in \mathcal{Q}} \sup _{g \in \mathcal{C}(x)} G(Z, \mathrm{E}[Z U(g)]),
$$

which combined with Lemma 4.6 yields the result.

Remark 4.7 For variational preferences, the convexity and concavity in the first and second argument, respectively, of the mapping $(Z, g) \mapsto \Psi_{\varepsilon}(Z, g)$ implies that no continuity properties of $\Psi_{\varepsilon}(Z, \cdot)$ are required for obtaining (4.10); cf. [71]. Since the continuity of $u(x)$ for this case is an immediate consequence of its concavity and finiteness, Theorem 3.5 therefore holds if we impose only Assumptions 2.8 and 3.2 (the asymptotic elasticity assumption on $U$ may also be dropped). In particular, the additional assumptions imposed in [71] for this result, namely that $u_{\mathbb{Q}}(x)<\infty$ for some $\mathbb{Q} \in \mathcal{Q}_{e}$ and that $v(y ; x)<\infty$ implies $v_{\mathbb{Q}}(y)<\infty$ for some $\mathbb{Q} \in \mathcal{Q}_{e}$, are in fact not needed. We note, however, that although Theorem 3.5 implies that

$$
u(x)=\sup _{X \in \mathcal{X}(x)} \inf _{\mathbb{Q} \in \mathcal{Q}^{f}} G\left(\mathbb{Q}, \mathrm{E}^{\mathbb{Q}}\left[U\left(X_{T}\right)\right]\right)
$$


and the supremum on the right-hand side is always attained, unless the assumptions of Theorem 3.1 hold, it is still not clear whether the original problem admits a solution. Finally, we note that for functions that are only quasi-convex-concave, lower and upper semicontinuity, respectively, in both arguments is in fact necessary for the minimax identity to hold; see [74, Remark 3.6] and [52, Example 2] for counterexamples.

We next prove the existence of a dual optimizer. The argument ultimately relies on the quasiconvexity and lower semicontinuity of the dual objective function.

Proof of Proposition 3.4 Note first that we may replace without loss of generality the set $\mathcal{Y}(y)$ in (3.1) by the set $\mathcal{D}(y)$ defined above (4.2) and consider the associated problem. Let

$$
H(Z, h):=G(Z, x y+\mathrm{E}[Z V(h / Z)]), \quad Z \in \mathcal{Q}, h \in \mathcal{D}(y) .
$$

Recall that $v(x ; y)$ is finite by assumption and let $\left(\left(Z_{n}, h_{n}\right)\right) \subseteq \mathcal{Q} \times \mathcal{D}(y)$ be an optimizing sequence such that

$$
H\left(Z_{n}, h_{n}\right) \underset{n \rightarrow \infty}{\searrow} v(y ; x) .
$$

We now argue that there exist $c \geq 0$ and $t \in \mathbb{R}$ such that we may assume without loss of generality that $\left(Z_{n}\right) \subseteq \mathcal{Q}_{t}(c)$. To this end, recall that $Z V(h / Z)=0$ on $\{Z=0\}$ and that $\mathrm{E}[h] \leq y, h \in \mathcal{D}(y)$. Since $V$ is decreasing and convex, use of Jensen's inequality yields for all $Z \in \mathcal{Q}, h \in \mathcal{D}(y)$ that

$$
\mathrm{E}[Z V(h / Z)] \geq V\left(\mathrm{E}^{\mathbb{Q}}[h / Z]\right)=V\left(\mathrm{E}\left[h \mathbb{1}_{\{Z>0\}}\right]\right) \geq V(y) .
$$

In consequence, $\mathrm{E}[Z V(h / Z)]+x y \geq U(x)$ for $Z \in \mathcal{Q}, h \in \mathcal{D}(y)$. Since $G(Z, \cdot)$ is nondecreasing, it therefore follows from (4.12) that

$$
\limsup _{n \rightarrow \infty} G\left(Z_{n}, U(x)\right)<\infty
$$

In consequence, without loss of generality, $\left(Z_{n}\right) \subseteq \mathcal{Q}_{t}(c)=\{Z \in \mathcal{Q}: G(Z, t) \leq c\}$ with $t:=U(x)$ and $c:=1+\limsup _{n \rightarrow \infty} G\left(Z_{n}, U(x)\right) \vee 0$.

Next, since $\mathcal{Q}_{t}(c)$ is uniformly integrable, we obtain from the proof of [72, Lemma 3.7] that the mapping $(Z, h) \mapsto \mathrm{E}[Z V(h / Z)]$ is jointly lower semicontinuous with respect to convergence in probability on $\mathcal{Q}_{t}(c) \times \mathcal{D}(y)$. Recall that $G$ is jointly lower semicontinuous if we use the weak topology in the first coordinate. Using again the uniform integrability of $\mathcal{Q}_{t}(c)$, we thus obtain that $(Z, h) \mapsto H(Z, h)$ is jointly lower semicontinuous with respect to convergence in probability on $\mathcal{Q}_{t}(c) \times \mathcal{D}(y)$.

Note that the mapping $(Z, h) \mapsto \mathrm{E}[Z V(h / Z)]$ is convex due to the convexity of $(z, y) \mapsto z V(y / z)$. Since $G(Z, \cdot)$ is nondecreasing and $G$ is jointly quasiconvex, we therefore have that $(Z, h) \mapsto H(Z, h)$ is jointly quasiconvex. Indeed, for $Z_{t}=t Z_{0}+(1-t) Z_{1}$ and $h_{t}=t h_{0}+(1-t) h_{1}$, 


$$
\begin{aligned}
H\left(Z_{t}, h_{t}\right) & =G\left(Z_{t}, x y+\mathrm{E}\left[Z_{t} V\left(h_{t} / Z_{t}\right)\right]\right) \\
& \leq G\left(Z_{t}, x y+t \mathrm{E}\left[Z_{0} V\left(h_{0} / Z_{0}\right)\right]+(1-t) \mathrm{E}\left[Z_{1} V\left(h_{1} / Z_{1}\right)\right]\right) \\
& \leq H\left(Z_{0}, h_{0}\right) \vee H\left(Z_{1}, h_{1}\right) .
\end{aligned}
$$

We now apply Lemma A1.1 in [21] twice to obtain a sequence $\left(\tilde{Z}_{n}, \tilde{h}_{n}\right)$ in $\operatorname{conv}\left\{\left(Z_{n}, h_{n}\right),\left(Z_{n+1}, h_{n+1}\right), \ldots\right\}$ converging $\mathbb{P}$-a.s. to some $\left(Z_{0}, h_{0}\right)$. Since $\mathcal{Q}_{t}(c)$ and $\mathcal{D}(y)$ are both convex, $\left(\tilde{Z}_{n}, \tilde{h}_{n}\right)$ is in $\mathcal{Q}_{t}(c) \times \mathcal{D}(y)$. Hence from (4.2), we have $h_{0} \in \mathcal{D}(y)$, and since $\mathcal{Q}_{t}(c)$ is weakly compact due to Assumption 3.2, we also have $Z_{0} \in \mathcal{Q}_{t}(c)$. Further, use of the joint quasiconvexity of $H$ and (4.12) yields

$$
H\left(\tilde{Z}_{n}, \tilde{h}_{n}\right) \leq \sup _{k \geq n} H\left(Z_{k}, h_{k}\right)=H\left(Z_{n}, h_{n}\right),
$$

and in consequence also $\left(\left(\tilde{Z}_{n}, \tilde{h}_{n}\right)\right)$ is an optimizing sequence. Use of the lower semicontinuity of $H$ then yields

$$
H\left(Z_{0}, h_{0}\right) \leq \liminf _{n \rightarrow \infty} H\left(\tilde{Z}_{n}, \tilde{h}_{n}\right)=v(y) .
$$

In consequence, the optimum is attained for $\left(Z_{0}, h_{0}\right)$.

Finally, suppose that $\left(Z_{1}, h_{1}\right)$ is another optimal pair. Then for $t \in[0,1]$, let $Z_{t}=t Z_{1}+(1-t) Z_{0}$ and $h_{t}=t h_{1}+(1-t) h_{0}$. According to (4.13), we have $H\left(Z_{t}, h_{t}\right) \leq v(y)$. Hence also $\left(Z_{t}, h_{t}\right)$ is optimal. In consequence, a maximal solution may be constructed along the same lines as in [71, Lemma 4.3].

Next, we prove Theorem 3.6 and Proposition 3.7 which establish the existence of a saddle point and the link between the primal and dual solutions.

Proof of Theorem 3.6 Given a saddle point $\left(\bar{X}_{T}, \widehat{\mathbb{Q}}\right)$ for the primal problem, take $y^{*}>0$ to be the value attaining the infimum in (4.3) for $\widehat{\mathbb{Q}}$. Existence is ensured due to the properties of the auxiliary value functions, see Lemma 4.2; indeed, by using Assumption 2.8 and the properties (i) and (iv) of Definition 2.3, we deduce that, without loss of generality, $u_{\widehat{\mathbb{Q}}}(x)<\infty, x>0$. Then

$$
u(x)=G\left(\widehat{Z}, u_{\widehat{Z}}(x)\right)=G\left(\widehat{Z}, v_{\widehat{Z}}\left(y^{*}\right)+x y^{*}\right),
$$

and it follows that the infimum in (3.3) is attained for $y^{*}$. Conversely, suppose that (3.3) is attained for some $y^{*}>0$. According to Lemma 2.9 and Proposition 3.4, the dual problem at level $y^{*}$ then admits a solution $(\widehat{\mathbb{Q}}, \widehat{Y})$. Recall that according to Theorem 3.1, there exists a primal optimizer $\bar{X}_{T}$. Now, since $G(Z, \cdot)$ is nondecreasing,

$$
u(x)=v\left(y^{*} ; x\right)=G\left(\widehat{Z}, v_{\widehat{Z}}\left(y^{*}\right)+x y^{*}\right) \geq G\left(\widehat{Z}, u_{\widehat{Z}}(x)\right) \geq u(x),
$$

which thus must hold as equality. In consequence,

$$
\begin{aligned}
u(x)=G\left(\widehat{Z}, u_{\widehat{Z}}(x)\right) & \geq G\left(\widehat{Z}, \mathrm{E}\left[\widehat{Z} U\left(\bar{X}_{T}\right)\right]\right) \\
& \geq \inf _{Z \in \mathcal{Q}} G\left(Z, \mathrm{E}\left[Z U\left(\bar{X}_{T}\right)\right]\right)=u(x),
\end{aligned}
$$

which shows that $\left(\bar{X}_{T}, \widehat{\mathbb{Q}}\right)$ is a saddle point for the primal problem. 
Next, from the definition of $\mathcal{Y}(y)$, we have $\mathrm{E}\left[X_{T} Y_{T}\right] \leq x y$, for $X \in \mathcal{X}(x)$ and $Y \in \mathcal{Y}(y)$. Hence, given a minimizer $y^{*}>0$ to (3.3), a corresponding dual solution $(\widehat{\mathbb{Q}}, \widehat{Y})$ and a solution $\bar{X}_{T}$ to the primal problem, it follows that

$$
\begin{aligned}
G & \left(\widehat{Z}, \mathrm{E}\left[\widehat{Z} V\left(\widehat{Y}_{T} / \widehat{Z}\right)+\bar{X}_{T} \widehat{Y}_{T}\right]\right)-G\left(\widehat{Z}, \mathrm{E}\left[\widehat{Z} U\left(\bar{X}_{T}\right)\right]\right) \\
& \leq G\left(\widehat{Z}, \mathrm{E}\left[\widehat{Z} V\left(\widehat{Y}_{T} / \widehat{Z}\right)\right]+x y^{*}\right)-u(x) \\
& =v\left(y^{*} ; x\right)-u(x)=0 .
\end{aligned}
$$

In consequence, if $G(Z, \cdot)$ is strictly increasing,

$$
\mathrm{E}^{\widehat{\mathbb{Q}}}\left[V\left(\widehat{Y}_{T} / \widehat{Z}\right)+\bar{X}_{T} \widehat{Y}_{T} / \widehat{Z}\right] \leq \mathrm{E}^{\widehat{\mathbb{Q}}}\left[U\left(\bar{X}_{T}\right)\right] .
$$

Since $V(y)+x y \geq U(x)$ for $x, y>0$, this inequality implies that $\widehat{\mathbb{Q}}$-a.s., we must have $V\left(\widehat{Y}_{T} / \widehat{Z}\right)+\bar{X}_{T} \widehat{Y}_{T} / \widehat{Z}=U\left(\bar{X}_{T}\right)$, which implies (3.4).

Proof of Proposition 3.7 Recall that (3.2) holds. Hence, in order to verify the existence of a saddle point, it suffices to show that the infimum in (3.2) is attained. Let $\left(Z_{n}\right) \subseteq \mathcal{Q}$ be an optimizing sequence such that

$$
G\left(Z_{n}, u_{Z_{n}}(x)\right) \searrow u(x) .
$$

Since $u_{Z_{n}}(x) \geq U(x), n \in \mathbb{N}$, and $G(Z, \cdot)$ is increasing, it follows that

$$
\limsup _{n \rightarrow \infty} G\left(Z_{n}, U(x)\right)<\infty .
$$

Hence without loss of generality, we may assume that $\left(Z_{n}\right) \subseteq \mathcal{Q}_{t}(c)$, where we choose $t:=U(x)$ and $c:=1+\limsup _{n \rightarrow \infty} G\left(Z_{n}, U(x)\right) \vee 0$. Recall that $\mathcal{Q}_{t}(c)$ is weakly compact. Since the pointwise supremum preserves lower semicontinuity and quasiconvexity, it follows by use of the same arguments as used to derive the quasiconvexity and lower semicontinuity of the mapping (4.9) that

$$
Z \mapsto G\left(Z, u_{Z}(x)\right)=\sup _{g \in \mathcal{C}(x)} G(Z, \mathrm{E}[Z U(g)])
$$

is quasiconvex and lower semicontinuous on $\mathcal{Q}_{t}(c)$ for convergence in probability. We now pick a sequence $\tilde{Z}_{n} \in \operatorname{conv}\left(Z_{n}, Z_{n+1}, \ldots\right)$ converging $\mathbb{P}$-a.s. to some $Z_{0}$; cf. [21, Lemma A1.1]. Since $\mathcal{Q}_{t}(c)$ is convex, we have $\left(\tilde{Z}_{n}\right) \subseteq \mathcal{Q}_{t}(c)$. Moreover, $\mathcal{Q}_{t}(c)$ is weakly compact due to Assumption 3.2. Hence, $Z_{0} \in \mathcal{Q}_{t}(c)$. By use of the same arguments as used in the proof of Proposition 3.4, the quasiconvexity and lower semicontinuity of the mapping (4.14) then yields that also $\left(\tilde{Z}_{n}\right)_{n \in \mathbb{N}}$ is an optimizing sequence, and in turn that $Z_{0}$ attains the infimum in (3.2).

Acknowledgements Open access funding provided by TU Wien (TUW). The author would like to thank two anonymous referees for helpful comments and suggestions and Michael Kupper, Martin Larsson and Alex Schied for valuable discussions.

Open Access This article is distributed under the terms of the Creative Commons Attribution 4.0 International License (http://creativecommons.org/licenses/by/4.0/), which permits unrestricted use, distribution, and reproduction in any medium, provided you give appropriate credit to the original author(s) and the source, provide a link to the Creative Commons license, and indicate if changes were made. 


\section{Appendix}

Proof of Lemma 4.2 The assumption $u_{Z}(x)<\infty$ implies that the expectation operator is defined in the standard way (cf. before Problem 2.6). Further, without loss of generality, the set of optimization objects in $u_{Z}(x)$ and $v_{Z}(y)$ may, respectively, be replaced by the sets $\mathcal{C}(x)$ and $\mathcal{D}(y)$ defined above (4.2).

Let $F_{n}(z, y)=\sup _{0<x \leq n}(z U(x)-x y)$ for $z \geq 0, y>0$. Further, define the set $\mathcal{B}_{n}:=\left\{g \in L_{+}^{0}: 0 \leq g \leq n\right\}$. Then $Z U(g)-g h \leq F_{n}(Z, h)$ a.s. for all $g \in \mathcal{B}_{n}$, $h \in \mathcal{D}(y)$, with the convention $0 \cdot \infty=0$. Moreover, for each $h \in \mathcal{D}(y)$, we may pick a sequence $\left(g_{k}\right)_{k \in \mathbb{N}}$ in $\mathcal{B}_{n}$ such that $Z U\left(g_{k}\right)-g_{k} h \nearrow F_{n}(Z, h)$ a.s. By the monotone convergence theorem, we therefore obtain

$$
\sup _{g \in \mathcal{B}_{n}} \mathrm{E}[Z U(g)-g h] \geq \lim _{k \rightarrow \infty} \mathrm{E}\left[Z U\left(g_{k}\right)-g_{k} h\right]=\mathrm{E}\left[F_{n}(Z, h)\right],
$$

which thus must hold as equality. Further, with $V_{n}(y)=\sup _{0<x \leq n}(U(x)-x y)$, we have

$$
F_{n}(z, y)= \begin{cases}0, & \text { for } z=0 \\ z F_{n}\left(1, \frac{y}{z}\right)=z V_{n}\left(\frac{y}{z}\right), & \text { for } z>0 .\end{cases}
$$

With the usual convention $Z V_{n}(h / Z):=0$ on $\{Z=0\}, h \in \mathcal{D}(y)$, we thus obtain

$$
\inf _{h \in \mathcal{D}(y)} \sup _{g \in \mathcal{B}_{n}} \mathrm{E}[Z U(g)-g h]=\inf _{h \in \mathcal{D}(y)} \mathrm{E}\left[Z V_{n}(h / Z)\right] .
$$

The first conjugacy relation in (4.3) now follows if it can be shown that as $n$ tends to $\infty$, the left- and right-hand sides of (A.1) converge to $\sup _{x>0}\left(u_{Z}(x)-x y\right)$ and $v_{Z}(y)$, respectively. As for the left-hand side, this follows exactly as in [50, Lemma 3.4]. Specifically, the argument consists in applying a minimax theorem and exploiting the duality between the sets $\mathcal{C}(x)$ and $\mathcal{D}(y)$ (cf. (4.2)), which remains unaltered by the modification of the utility function. As for the right-hand side, the argument of Lemma 3.4 in [50] goes through as long as we can ensure the uniform integrability of the set $\left\{Z V_{n}^{-}\left(h_{n} / Z\right): n \in \mathbb{N}\right\}$ for $\left(h_{n}\right) \subseteq \mathcal{D}(y)$. To this end, we now argue as follows. Note that for $I(y) \leq n$, it holds that $V_{n}(y)=V(y)$. As $V_{n}^{-}$is increasing in $y$ and decreasing in $n$, it follows that

$$
Z V_{n}^{-}\left(h_{n} / Z\right) \leq Z V^{-}\left(h_{n} / Z\right)+Z V_{1}^{-}\left(U^{\prime}(1)\right)
$$

Recall that $Z$ is integrable. In consequence, according to [72, Lemma 3.6], the family $\left\{Z V^{-}(h / Z): h \in \mathcal{D}(y)\right\}$ is uniformly integrable. Hence, it follows from (A.2) that so is $\left\{Z V_{n}^{-}\left(h_{n} / Z\right): n \in \mathbb{N}\right\}$.

The second conjugacy relation in (4.3) follows directly from the first since $u_{\mathbb{Q}}(x)$ is finite and concave (cf. e.g. [69, Theorem 12.2]).

Next, since $A E(U(\cdot))<1$, we may apply Lemma 6.3 in [50] to obtain $\alpha, \beta, \gamma>0$ such that $z V\left(\frac{y / 2}{z}\right) \leq \alpha z V\left(\frac{y}{z}\right)+\beta y+\gamma z$ for all $y, z>0$. Combined with the finiteness of $u_{Z}(x), x>0$, and the conjugacy relations (4.3), this yields the finiteness of $v_{Z}(y)$, $y>0$; cf. Note 2 in [51]. In turn, as argued in Note 1 in [51], again due to the 
conjugacy relations between $u_{Z}$ and $v_{Z}$, the finiteness of $v_{Z}(y), y>0$, is equivalent to

$$
\lim _{x \nearrow \infty} \frac{u_{Z}(x)}{x}=0
$$

The $\mathbb{Q}$-uniform integrability of $\left\{U^{+}\left(g_{n}\right): n \in \mathbb{N}\right\}$ may therefore be established following the proof of Lemma 1 in [51]. Properties (4.4) and (4.5) then follow by use of the same arguments as in [50].

\section{References}

1. Acciaio, B., Goldammer, V.: Optimal portfolio selection via conditional convex risk measures on $L^{p}$. Decis. Econ. Finance 36, 1-21 (2013)

2. Anderson, E.W., Hansen, L.P., Sargent, T.J.: A quartet of semigroups for model specification, robustness, prices of risk, and model detection. J. Eur. Econ. Assoc. 1, 68-123 (2003)

3. Artzner, P., Delbaen, F., Eber, J.-M., Heath, D.: Coherent measures of risk. Math. Finance 9, 203-228 (1999)

4. Backhoff Veraguas, J.D., Fontbona, J.: Robust utility maximization without model compactness. SIAM J. Financ. Math. 7, 70-103 (2016)

5. Biagini, S., Bion-Nadal, J.: Dynamic quasi concave performance measures. J. Math. Econ. 55, 143153 (2014)

6. Bion-Nadal, J.: Time consistent dynamic risk processes. Stoch. Process. Appl. 119, 633-654 (2009)

7. Björk, T., Murgoci, A., Zhou, X.Y.: Mean-variance portfolio optimization with state-dependent risk aversion. Math. Finance 24, 1-24 (2014)

8. Bordigoni, G., Matoussi, A., Schweizer, M.: A stochastic control approach to a robust utility maximization problem. In: Benth, F.E., et al. (eds.) Stochastic Analysis and Applications. Abel Symp., vol. 2, pp. 125-151. Springer, Berlin (2007)

9. Burgert, C., Rüschendorf, L.: Optimal consumption strategies under model uncertainty. Stat. Decis. 23, 1-14 (2005)

10. Cerreia-Vioglio, S., Ghirardato, P., Maccheroni, F., Marinacci, M., Siniscalchi, M.: Rational preferences under ambiguity. Econom. Theory 48, 341-375 (2011)

11. Cerreia-Vioglio, S., Maccheroni, F., Marinacci, M., Montrucchio, L.: Uncertainty averse preferences. J. Econ. Theory 146, 1275-1330 (2011)

12. Cerreia-Vioglio, S., Maccheroni, F., Marinacci, M., Montrucchio, L.: Complete monotone quasiconcave duality. Math. Oper. Res. 36, 321-339 (2011)

13. Cerreia-Vioglio, S., Maccheroni, F., Marinacci, M., Montrucchio, L.: Risk measures: rationality and diversification. Math. Finance 21, 743-774 (2011)

14. Chateauneuf, A., Faro, J.H.: Ambiguity through confidence functions. J. Math. Econ. 45, 535-558 (2009)

15. Chen, Z., Epstein, L.: Ambiguity, risk, and asset returns in continuous time. Econometrica 70, 1403$1443(2002)$

16. Cox, J.C., Huang, C.: Optimal consumption and portfolio policies when asset prices follow a diffusion process. J. Econ. Theory 49, 33-83 (1989)

17. Czichowsky, C.: Time-consistent mean-variance portfolio selection in discrete and continuous time. Finance Stoch. 17, 227-271 (2013)

18. Debreu, G.: Theory of Value. Yale University Press, New Haven (1959)

19. Delbaen, F.: Coherent risk measures on general probability spaces. In: Sandmann, K., Schönbucher, P.J. (eds.) Advances in Finance and Stochastics. Essays in Honour of Dieter Sondermann, pp. 1-37. Springer, Berlin (2002)

20. Delbaen, F., Peng, S., Rosazza Gianin, E.: Representation of the penalty term of dynamic concave utilities. Finance Stoch. 14, 449-472 (2010)

21. Delbaen, F., Schachermayer, W.: A general version of the fundamental theorem of asset pricing. Math. Ann. 300, 463-520 (1994)

22. Delbaen, F., Schachermayer, W.: The fundamental theorem of asset pricing for unbounded stochastic processes. Math. Ann. 312, 215-250 (1998) 
23. Denis, L., Kervarec, M.: Optimal investment under model uncertainty in nondominated models. SIAM J. Control Optim. 51, 1803-1822 (2013)

24. Drapeau, S., Kupper, M.: Risk preferences and their robust representation. Math. Oper. Res. 38, 28-62 (2013)

25. Ellsberg, D.: Risk, ambiguity, and the Savage axioms. Q. J. Econ. 75(4), 643-669 (1961)

26. Faidi, W., Matoussi, A., Mnif, M.: Maximization of recursive utilities: a dynamic maximum principle approach. SIAM J. Financ. Math. 2, 1014-1041 (2011)

27. Föllmer, H., Leukert, P.: Efficient hedging: cost versus shortfall risk. Finance Stoch. 4, 117-146 (2000)

28. Föllmer, H., Penner, I.: Convex risk measures and the dynamics of their penalty functions. Stat. Decis. 24, 61-96 (2006)

29. Föllmer, H., Schied, A.: Convex measures of risk and trading constraints. Finance Stoch. 6, 429-447 (2002)

30. Föllmer, H., Schied, A.: Stochastic Finance. An Introduction in Discrete Time, 2nd edn. De Gruyter Studies in Mathematics, vol. 27. de Gruyter, Berlin (2004)

31. Föllmer, H., Schied, A., Weber, S.: Robust preferences and robust portfolio choice. In: Bensoussan, A., Zhang, Q. (eds.) Handbook of Numerical Analysis. Mathematical Modeling and Numerical Methods in Finance, vol. 15, pp. 29-87. Elsevier, Amsterdam (2009)

32. Frittelli, M., Maggis, M.: Dual representation of quasi-convex conditional maps. SIAM J. Financ. Math. 2, 357-382 (2011)

33. Frittelli, M., Maggis, M.: Complete duality for quasiconvex dynamic risk measures on modules of the $L^{p}$-type. Stat. Risk. Model. 31, 103-128 (2014)

34. Frittelli, M., Rosazza Gianin, E.: Putting order in risk measures. J. Bank. Finance 26, 1473-1486 (2002)

35. Gilboa, I., Schmeidler, D.: Maxmin expected utility with nonunique prior. J. Math. Econ. 18, 141-153 (1989)

36. Hansen, L.P., Sargent, T.J.: Robust control and model uncertainty. Am. Econ. Rev. 91, 60-66 (2001)

37. Hernández-Hernández, D., Schied, A.: Robust utility maximization in a stochastic factor model. Stat. Decis. 24, 109-125 (2006)

38. Hernández-Hernández, D., Schied, A.: A control approach to robust utility maximization with logarithmic utility and time-consistent penalties. Stoch. Process. Appl. 117, 980-1000 (2007)

39. Hernández-Hernández, D., Schied, A.: Robust maximization of consumption with logarithmic utility. In: Proceedings of the 2007 American Control Conference, pp. 1120-1123. IEEE Press, New York (2007)

40. Jeanblanc, M., Matoussi, A., Ngoupeyou, A.: Robust utility maximization problem in a discontinuous filtration (2012). arXiv:1201.2690

41. Kahneman, D., Tversky, A.: Prospect theory: an analysis of decision under risk. Econometrica 47(2), 263-291 (1979)

42. Källblad, S.: Topics in Portfolio Choice: Qualitative Properties, Time Consistency and Investment under Model Uncertainty. DPhil Thesis, University of Oxford: Mathematical Institute (2014). Available online at https://papers.ssrn.com/sol3/papers.cfm?abstract_id=2523688

43. Källblad, S., Obłój, J., Zariphopoulou, T.: Time-consistent investment under model uncertainty: the robust forward criteria (2013). arXiv:1311.3529

44. Karatzas, I., Kardaras, C.: The numéraire portfolio in semimartingale financial models. Finance Stoch. 11, 447-493 (2007)

45. Karatzas, I., Lehoczky, J.P., Shreve, S.E.: Optimal portfolio and consumption decisions for a "small investor" on a finite horizon. SIAM J. Control Optim. 25, 1557-1586 (1987)

46. Karatzas, I., Lehoczky, J.P., Shreve, S.E., Xu, G.-L.: Martingale and duality methods for utility maximization in an incomplete market. SIAM J. Control Optim. 29, 702-730 (1991)

47. Klibanoff, P., Marinacci, M., Mukerji, S.: A smooth model of decision making under ambiguity. Econometrica 73, 1849-1892 (2005)

48. Klöppel, S., Schweizer, M.: Dynamic indifference valuation via convex risk measures. Math. Finance 17, 599-627 (2007)

49. Knight, F.H.: Risk, Uncertainty and Profit. Hart, Schaffner and Marx, New York (1921)

50. Kramkov, D., Schachermayer, W.: The asymptotic elasticity of utility functions and optimal investment in incomplete markets. Ann. Appl. Probab. 9, 904-950 (1999)

51. Kramkov, D., Schachermayer, W.: Necessary and sufficient conditions in the problem of optimal investment in incomplete markets. Ann. Appl. Probab. 13, 1504-1516 (2003) 
52. Kim, W.K., Kum, S.: On a non-compact generalization of Fan's minimax theorem. Taiwan. J. Math. 14, 347-358 (2010)

53. Laeven, R.J.A., Stadje, M.: Robust portfolio choice and indifference valuation. Math. Oper. Res. 39, 1109-1141 (2014)

54. Larsen, K., Žitković, G.: Stability of utility-maximization in incomplete markets. Stoch. Process. Appl. 117, 1642-1662 (2007)

55. Larsson, M.: Non-equivalent beliefs and subjective equilibrium bubbles. (2013). arXiv:1306.5082

56. Lazrak, A., Quenez, M.C.: A generalized stochastic differential utility. Math. Oper. Res. 28, 154-180 (2003)

57. Maccheroni, F., Marinacci, M., Rustichini, A.: Ambiguity aversion, robustness, and the variational representation of preferences. Econometrica 74, 1447-1498 (2006)

58. Mastrogiacomo, E., Rosazza Gianin, E.: Pareto optimal allocations and optimal risk sharing for quasiconvex risk measures. Math. Financ. Econ. 9, 149-167 (2015)

59. Mastrogiacomo, E., Rosazza Gianin, E.: Portfolio optimization with quasiconvex risk measures. Math. Oper. Res. 40, 1042-1059 (2015)

60. Merton, R.C.: Lifetime portfolio selection under uncertainty: the continuous-time case. Rev. Econ. Stat. 51(3), 247-257 (1969)

61. Merton, R.C.: Optimum consumption and portfolio rules in a continuous-time model. J. Econ. Theory 3, 373-413 (1971)

62. Mostovyi, O.: Necessary and sufficient conditions in the problem of optimal investment with intermediate consumption. Finance Stoch. 19, 135-159 (2015)

63. Müller, M.: Market Completion and Robust Utility Maximization. PhD thesis, Humboldt-Universität zu Berlin, Mathematisch-Naturwissenschaftliche Fakultät II (2005). Available online at http://edoc. hu-berlin.de/docviews/abstract.php?lang=ger\&id=26287

64. Nutz, M.: Utility maximization under model uncertainty in discrete time. Math. Finance 26, 252-268 (2016)

65. Owari, K.: Robust exponential hedging in a Brownian setting. JSIAM Lett. 1, 64-67 (2009)

66. Peng, S.: Dynamically consistent nonlinear evaluations and expectations (2005). arXiv:math/0501415

67. Pliska, S.R.: A stochastic calculus model of continuous trading: optimal portfolios. Math. Oper. Res. 11, 370-382 (1986)

68. Quenez, M.-C.: Optimal portfolio in a multiple-priors model. In: Dalang, R.C., et al. (eds.) Seminar on Stochastic Analysis, Random Fields and Applications IV. Progr. Probab., vol. 58, pp. 291-321. Birkhäuser, Basel (2004)

69. Rockafellar, R.T.: Convex Analysis. Princeton Mathematical Series, vol. 28. Princeton University Press, Princeton (1970)

70. Savage, L.J.: The Foundations of Statistics. Wiley, New York (1954)

71. Schied, A.: Optimal investments for risk- and ambiguity-averse preferences: a duality approach. Finance Stoch. 11, 107-129 (2007)

72. Schied, A., Wu, C.-T.: Duality theory for optimal investments under model uncertainty. Stat. Decis. 23, 199-217 (2005)

73. Schmeidler, D.: Subjective probability and expected utility without additivity. Econometrica 57, 571587 (1989)

74. Sion, M.: On general minimax theorems. Pac. J. Math. 8, 171-176 (1958)

75. Skiadas, C.: Robust control and recursive utility. Finance Stoch. 7, 475-489 (2003)

76. Strzalecki, T.: Axiomatic foundations of multiplier preferences. Econometrica 79, 47-73 (2011)

77. von Neumann, J., Morgenstern, O.: Theory of Games and Economic Behavior, 2nd edn. Princeton University Press, Princeton (1947)

78. Weston, K.: Stability of utility maximization in nonequivalent markets. Finance Stoch. 20, 511-541 (2016)

79. Wittmüss, W.: Robust optimization of consumption with random endowment. Stochastics 80, 459475 (2008) 